\title{
An Alternative to Dark Matter? Part 3: An Open Universe (3 Gy to 76 Gy) Galaxies and Structures Rotation
}

\author{
Jean Perron (1) \\ Department of Applied Sciences, UQAC, 555 boul Université, Chicoutimi, Canada \\ Email: jean_perron@uqac.ca
}

How to cite this paper: Perron, J. (2021) An Alternative to Dark Matter? Part 3: An Open Universe (3 Gy to 76 Gy) Galaxies and Structures Rotation. Journal of High Energy Physics, Gravitation and Cosmology, 7, 844-872.

https://doi.org/10.4236/jhepgc.2021.73048

Received: March 17, 2021

Accepted: June 22, 2021

Published: June 25, 2021

Copyright (c) 2021 by author(s) and Scientific Research Publishing Inc. This work is licensed under the Creative Commons Attribution International License (CC BY 4.0).

http://creativecommons.org/licenses/by/4.0/

\begin{abstract}
A cosmological model was developed using the equation of state of photon gas, as well as cosmic time. The primary objective of this model is to see if determining the observed rotation speed of galactic matter is possible, without using dark matter (halo) as a parameter. To do so, a numerical application of the evolution of variables in accordance with cosmic time and a new state equation was developed to determine precise, realistic values for a number of cosmological parameters, such as energy of the universe $U$, cosmological constant $\Lambda$, curvature of space $k$, energy density $\rho_{\Lambda e}$, age of the universe $t_{\Omega}$ (part 1). That energy of the universe, when taken into consideration during the formation of the first galaxies ( $<1[\mathrm{~Gy}])$, provides a relatively adequate explanation of the non-Keplerian rotation of galactic masses (part 2). Indeed, such residual, non-baryonic energy, when considered in Newton's gravity equation, adds the term $F_{\Lambda}(r)$, which can partially explain, without recourse to dark matter, the rotations of some galaxies, such as M33, UGC12591, UGC2885, NGC3198, NGC253, DDO161, UDG44, the MW and the Coma cluster. Today, in the MW, that cosmological gravity force is in the order of $10^{26}$ times smaller than the conventional gravity force. The model predicts an acceleration of the mass in the universe $(q \sim-0.986)$; the energy associated with curvature $E_{k}$ is the driving force behind the expansion of the universe, rather than the energy associated with the cosmological constant $E_{\Lambda}$. An equation to determine expansion is obtained using the energy form of the Friedmann equation relative to Planck power $P_{P}$ and cosmic time or Planck force $F_{P}$ acting at the frontier of the universe moving at $c$. This constant Planck force, from unknown sources, acts everywhere to the expansion of the universe as a stretching effect on the volume. Finally, the model partly explains the value $\mathrm{a}_{0}$ of the MOND theory. Indeed, $a_{0}$ is not a true constant, but depends on the cosmological constant at the time the great structures were
\end{abstract}


formed ( 1 [Gy]), as well as an adjustment of the typical mass and dimension of those great structures, such as galaxies. The constant $\mathrm{a}_{0}$ is a different expression of the cosmological gravity force $F_{\Lambda}$ as expressed by the cosmological constant, $\Lambda$, acting through the energy-mass equivalent during the formation of the structures. It does not put in question the value of $G$.

\section{Keywords}

Galaxies Kinematic and Dynamic, Galaxies Coma Cluster, Galaxies Evolution

\section{Attractive Cosmological Gravity, $F_{\Lambda}$, and Galaxy Rotation (Simplified Model)}

The formation and evolution of galaxies is a very complex field of study, and the associated mechanisms have not yet been fully interpreted. Indeed, the number of phenomena in play during galactogenesis, such as supplemental forces to gravity, the birth of stars and internal structures, energy dissipation effects, and the quantity and type of neighbouring matter being absorbed are only some of the factors involved in galaxy formation [1]. A relatively complete model has been put forth by [2] Martig et al., which assumes the presence or existence of dark matter that is as much subject to gravity (Kepler) as baryonic matter. In this article, as aforementioned, we do not consider the existence of dark matter, but rather energy at time $t$ (non-massive) and the mass-energy equivalence acting through the cosmological constant. This has already been discussed by [3] Gessner, where the cosmological constant did not vary during the formation process of the structures. This may be due to the lack of a predictive model for $\Lambda(t)$, which hinders the simulation of velocity profiles for structures with either small or great radii. With such a predictive model, the impact of this attractive force on galaxy rotation can be seen. We do know the values of the cosmological constant, $\Lambda$, at the time of primitive galaxy formation (1 - 2 [Gy]). We can calculate that attractive force and see its effects on the rotation of some galaxies. Put simply, for a given circular rotation orbit, the tangential rotation speed of a mass is expressed through the balance of the main forces considered in the model: gravity and cosmological gravity via mass-energy equivalence:

$$
\begin{gathered}
F_{c}=F_{G}+F_{\Lambda} \\
m \omega^{2} r=G m\left(\frac{M}{r^{2}}+\frac{\Lambda c^{2} r}{6 G}\right) \\
v_{t}^{2}=G\left(\frac{M}{r}+\frac{\Lambda c^{2} r^{2}}{6 G}\right)
\end{gathered}
$$

Note that cosmological attractive force associated with $\Lambda$ is supplemental to conventional gravity (baryonic). Moreover, that force cannot be attributed to negative masses or so-called "dark" unobservable forces. In fact, the denomina- 
tor of the second term is not the inverse of the radius, which confirms that the force is not due to the effects of mass as such, but to a mass-energy equivalence associated with $\Lambda$. Finally, because that force is relative to $\Lambda$, which is relative to the age of the universe, the rotation profile of masses like galaxies is in turn relative to time from the standpoint of forces in play. In other words, the rotation profile should take into consideration the evolution of $\Lambda$ as the galaxy absorbs matter over time. The actual process behind the action of this cosmological gravity, $F_{\Lambda}$, on the rotation dynamics of galaxies is complex, as it is relative to both time and the radius of any given galaxy:

$$
v_{t}^{2}(r, t)=G\left(\frac{\rho(r, t) V(r, t)}{r(t)}+\frac{\Lambda(t) c^{2} r(t)^{2}}{6 G}\right)
$$

Solving this equation is beyond the scope of this paper because we would need to know the density profile of matter in the galaxy relative to time, $t$, meaning the formation mechanismes of that galaxy from a dynamic standpoint (mass accumulation process and rate). A simulator like Millenium could derive that term associated with $F_{\Lambda}$. However, in this paper, we want to demonstrate that assuming the existence of dark matter is not necessary at first to describe the galaxy formation process and rotation curves as we see them today. To do so, the galaxy formation process can be simplified by assuming that mass accumulates according to a simple function of time, and that $\Lambda(t)$ also evolves according to time (bottom-up model). The simplified equation of galaxy rotation has three terms, the effects associated with the bulbe, or denser central area, and with the disc around the central area, and the effects of $\Lambda(t)$ at formation time $t$ and radius $r(t)$. At first, we will not consider dark matter, also called halo mass, although such dark baryonic mass (non-radiating) surely must exist within galaxies. We will see that for some galaxies, such as M33, the observable mass (luminous) is not sufficient to explain the observed rotations, meaning that we have to assume the probable existence of baryonic dark masses.

The time at which a galaxy started to form is important because it influences the effective value of $\Lambda$. Then, the formation time of the galaxy is just as important (acceleration rate of the mass), since this yields the total variation of $\Lambda$ on the rotation process. To initially demonstrate the effects of force $F_{\Lambda}$ on galaxy rotation, let us find an expression of rotation speed relative to time: the time at which the galaxy started to form, $t_{i}$, the total formation time of the galaxy, $t_{T}$, with variable force, $F_{\Lambda}$, acting during that formation time, $t_{T}-t_{i}$. For the masses of the bulbe and disc, we get a simplified expression:

$$
\begin{gathered}
M_{T}(r, t)=M_{b}(r, t)+M_{d}(r, t) \\
M_{T}(r, t)=\frac{V_{b}(r, t)}{V_{b_{T}}} M_{b}+\frac{V_{d}(r, t)}{V_{d_{T}}} M_{d}=\frac{r(t)^{3}}{r_{b}^{3}} M_{b}+\frac{r(t)^{2}-r_{b}^{2}}{r_{T}^{2}-r_{b}^{2}} M_{d}
\end{gathered}
$$

where:

$$
0 \leq r(t) \leq r_{b}, \text { for the bulbe }
$$


$r_{b} \leq r(t) \leq r_{T}$, for the disc:

$r_{b}$ : bulbe radius determined at the end of galaxy formation;

$r_{T}$ : disc and bulbe radii determined at the end of galaxy formation;

$M_{b}$ : bulbe mass determined at the end of galaxy formation;

$M_{\dot{d}}$ disc mass determined at the end of galaxy formation.

A simple law can be used to calculate mass accumulation at a constant rate:

$$
r(t)=\alpha t=\frac{t}{t_{T}-t_{i}} r_{T}
$$

where,

$t_{i} \leq t \leq t_{T}$, formation time of the galaxy;

$\alpha$ : galaxy radius growth rate (accumulation).

For the mass, we get:

$$
M_{T}(r, t)=\frac{\alpha^{3} t^{3}}{r_{b}^{3}} M_{b}+\frac{\alpha^{2} t^{2}-r_{b}^{2}}{r_{T}^{2}-r_{b}^{2}} M_{d}
$$

For rotation speed, we get:

$$
\begin{gathered}
v_{t}^{2}=G\left(\frac{M}{r}+\frac{\Lambda c^{2} r^{2}}{6 G}\right)=\frac{G}{r}\left[\frac{\alpha^{3} t^{3}}{r_{b}^{3}} M_{b}+\frac{\alpha^{2} t^{2}-r_{b}^{2}}{r_{T}^{2}-r_{b}^{2}} M_{d}\right]+\frac{k_{\Lambda} c^{2} \alpha^{2} t^{2}}{6\left(t+t_{i}\right)^{4}} \\
v_{t}^{2}=G\left[\frac{\alpha^{2} t^{2}}{r_{b}^{3}} M_{b}+\frac{\alpha^{2} t^{2}-r_{b}^{2}}{\alpha t\left(r_{T}^{2}-r_{b}^{2}\right)} M_{d}\right]+\frac{k_{\Lambda} c^{2} \alpha^{2} t^{2}}{6\left(t+t_{i}\right)^{4}}
\end{gathered}
$$

where,

$t_{i} \leq t \leq t_{T}$ : formation time of the galaxy;

$t_{i} \leq t_{b} \leq t_{B}$ : formation time of the galaxy bulbe;

$t_{B} \leq t_{d} \leq t_{T}$ : formation time of the galaxy disc.

$t_{i}$ : age of the universe at the time of galaxy formation to calculate $\Lambda(t)=\frac{k_{\Lambda}}{\left(t_{i}+t\right)^{4}}=k_{\Lambda} H^{4}$, with:

$$
k_{\Lambda}=\left[\frac{32 \pi G \sigma C_{1}^{4}}{c^{5}}\right]=\left[\frac{32 \pi G \sigma T_{\Omega}^{4} t_{\Omega}^{4}}{c^{5}}\right]=2.88 \times 10^{17}\left[\mathrm{~s}^{4} \cdot \mathrm{m}^{-2}\right]
$$

In the above equation for $v_{t}$, the first term is for the attraction of the bulbe on the rotating mass, the second, for the attraction of the disc, and the third, for the attraction of force $F_{\Lambda}$ due to the cosmological constant through the residual mass-energy equivalence of the universe at the beginning of formation, $t_{i}$, of the galaxy acting throughout formation time, $t_{T}-t_{i}$. This equation contains the essential elements for predicting the rotation curve of the luminous mass of galaxies. Force $F_{\Lambda}$ decreases over time, or the age of the universe, but one must consider that the prevailing conditions of galaxy formation are still present in the space-time continuum of that galaxy. In other words, we will see that, in simulations of the rotation of some galaxies, the time at which mass started to accumulate is crucial for the development of the type of rotation because cosmological gravity varies like $t^{t}$, or inversely with the age of the universe during the forma- 
tion of that galaxy. This means that the type of rotation curve (concave or convex) lets us know, in part, whether the galaxy was formed in the early days of the universe, or later (concave $=$ older; convex $=$ younger). So, a weaker cosmological gravity should lead to Keplerian rotation, or a convex curve. Finally, we have assumed a very simple galaxy radius growth rate that is linear over time. Other, more realistic models can be introduced in the equation to better illustrate the generic growth of an isolated galaxy. Of course, the impacts of galactic collisions and agglomerations are not considered here.

\section{Mass Rotation Equation and Tangential Velocity}

The rotation equation involves five parameters to determine, at first glance, the rotation of a galaxy assuming that it has not undergone severe transformations, such as collisions with other massive bodies. In this study, we propose a bottom-up approach with the following parameters:

- Start time of bulbe formation (beginning of accumulation);

- Galaxy formation time (end of major accumulation);

- Formation time of the bulbe (the disc begins to form);

- Bulbe mass accumulated during bulbe formation time;

- Disc mass accumulated during disc formation time.

The actual mass distribution and radial velocity of galaxies are complex, other parameters have to be considered, such as the presence of gases, and small neighbouring structures or more massive structures nearby (like other galaxies), etc. However, we will see that the equation requires careful consideration to significantly reduce the need to consider the dark matter halo (invisible) to explain rotation speeds. Dark matter is not considered in this model, but we do consider non-luminous baryonic matter.

\section{MW, S(B)bc I-II}

Many studies have been conducted to try and determine the velocity profile and mass of the MW. Several variations of the luminous mass have been reported, and many authors include an estimation of the dark matter halo to validate certain observations or conclusions. Indeed, according to a number of studies, the total mass of the MW can vary by as much as a factor of seven ( $\left.5.8 \times 10^{11} M_{\odot}<M_{t}<4.5 \times 10^{12} M_{\odot}\right)$ [4] [5], depending on the presence or absence of dark matter. Moreover, the accepted structure of the MW includes a $~ 1$ [kpc] (luminous mass of $1.7 \times 10^{10} M_{\odot}$ ) center with radio emissions, a thick disc of $\sim 1$ [kpc] (luminous mass of $1 \times 10^{9} M_{\odot}$ ), a thin disc of $\sim 20$ [kpc] (luminous mass of $6 \times 10^{10} M_{\odot}$ ), and several spiral arms. Also, the MW may have collided with Andromeda in the past, but this is an unverified assumption. In other words, the velocity profile of the MW may have been disrupted by past events that the velocity prediction model cannot consider due to a lack of relevant data.

Figure 1 and Figure 2 show an approximate evolution for the mean rotation curve of the MW from the time formation began $\left(t_{i}=0.181\right.$ [Gy] and $t_{t}=0.32$ 
[Gy]) until today ( $t_{\Omega}=76.1[\mathrm{~Gy}]$ ). The galaxy's mass is considered constant after its main formation. Realistically, however, accumulation is a continuous process. In this model, we will see that the main formation of galaxies seems to have occurred around the beginning of the universe $(<1.5[\mathrm{~Gy}])$, and that accumulation progressively decreases thereafter, even though the intrinsic motion of galaxies continues over time and events (collisions, restructurings, amalgamations). In fact, the early formation of structures like massive black holes and galaxies $(<500$ [My]) could be made possible by a direct collapse mechanism [6] [7]. Recently, a team discovered a candidate galaxy, SPT0615-JD, at $z \sim 10-11$, that may have existed around 400 [My] after the beginning [8]. Also, another team reported the lens-effect observation of a star dating back to earlier than 250 [My] in galaxy MACS1149-JD1 [9]. Lately, the ALPINE-ALMA project to confirm the existence of 118 galaxies matures much earlier than was thought possible [10].

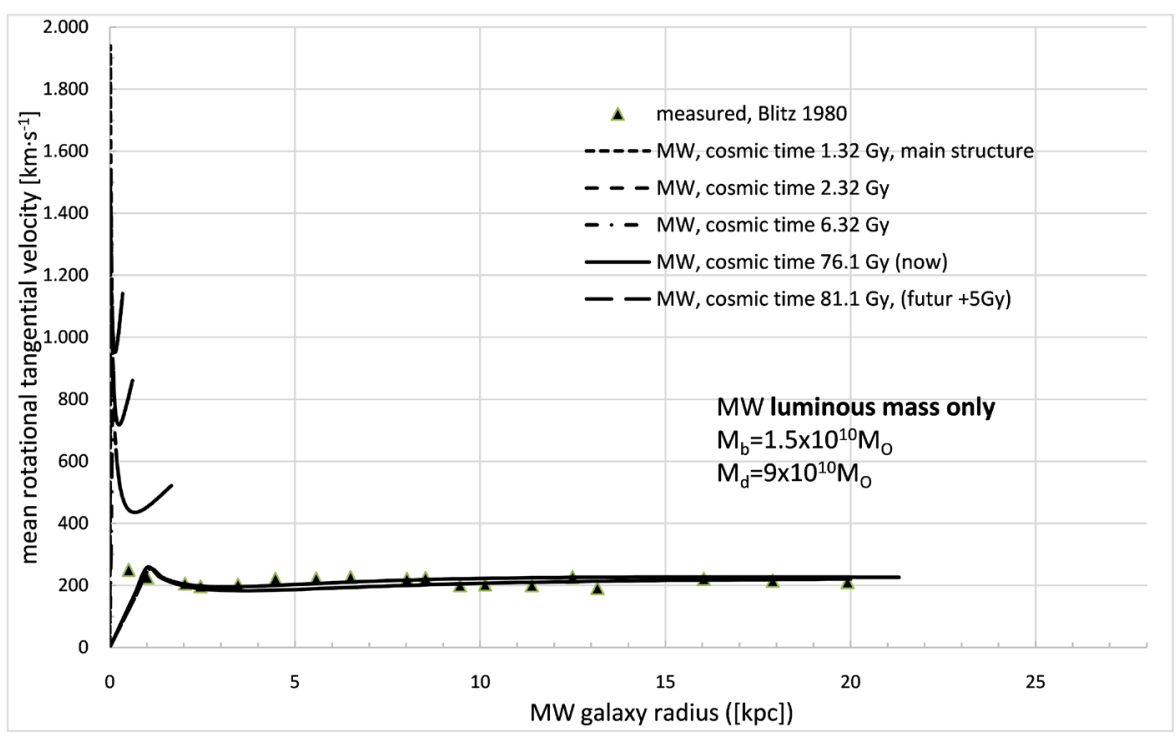

Figure 1. MW rotational and size evolution since formation to 81.1 [Gy].

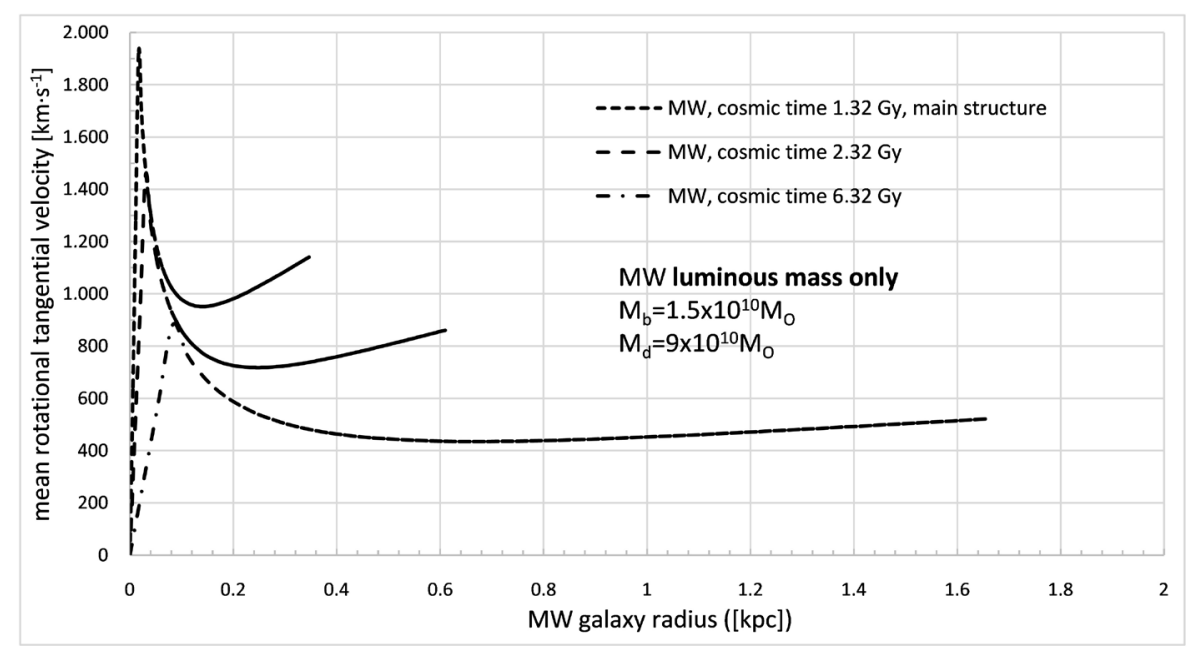

Figure 2. MW rotational and size evolution since formation to 6 [Gy]. 
The simulation process is as follows: The primitive formation of the galaxy is determined by trial and error using the aforementioned five-paremeter equation. Then, the galaxy undergoes expansion of the universe on a scale factor until today. The simulation can be extended into the MW's future. At the beginning, the MW had a radius of about $r=0.34$ [kpc]. This is smaller than stated by Martig et al. [2], a disc dimension derived from dynamic galaxy simulations $\left(r_{\text {core }} \sim 1.79\right.$ [kpc], case G10). However, taking into consideration the appropriate scale factor for the simulations by Martig $(z=2)(4.6[\mathrm{~Gy}] / 13.8$ [Gy]), the starting dimension of the galaxy disc for case G10 is reduced to $\sim 0.59$. Rotation speed of the center was quite high, around $1100\left[\mathrm{k} \cdot \mathrm{ms}^{-1}\right]$. Then, the MW increased in size by a scale factor and speeds dropped. Around 6 [Gy], its diameter was around 1.6 [kpc].

Figure 3 shows three velocity profiles [6] with observed masses and the three remaining parameters of the equation: $t_{p} t_{T}$ and $t_{b}$, along with cosmological gravity, $F_{\Lambda}$, calculated at the time the MW was formed. The first curve is observed masses and Kepler rotation speeds only. Note that the speeds for large radii are not well represented. The second curve is for a galaxy formation beginning earlier, around 150 [My], but the longer formation time of 450 [My]. Note that the speeds are better represented than in the previous graph but are generally still too low. The last curve is the formation of the MW, which may have begun around 180 [My], with the main accumulation lasting some 320 [My]. Here, the speeds do not follow the measured profile closely, but the values are in the proper order of magnitude for great radii. Note that speed variations, around 10 [kpc], are not well represented, which could indicate a much more complex accumulation process than the linear model used (change of rate). As concerns the beginning of formation, around 180 [My], observations have shown that stars like HE 1523-0901 and HD 140283 are as old as 600 [My] and even 150 [My] after the beginning [11]. HE 1523-0901 is located 7500 light years away from Earth (2.29 [kpc]). If we estimate the position of the sun to be $r=8.5 \pm 0.5[\mathrm{kpc}]$, the formation of a star at that position is possible:

$$
\begin{gathered}
t>t_{i}+\frac{r_{\odot} \pm 0.5}{\alpha} \pm \frac{2.29}{\alpha}=181[\mathrm{My}]+\frac{8.5 \pm 0.5 \mathrm{kpc}}{\frac{20 \mathrm{kpc}}{320 \mathrm{My}} \pm \frac{2.29 \mathrm{kpc}}{\frac{20 \mathrm{kpc}}{320 \mathrm{My}}}} \\
t=181+136 \pm 8[\mathrm{My}] \pm 37[\mathrm{My}]=317 \pm 45[\mathrm{My}]
\end{gathered}
$$

That star was formed about $500-600$ [My] after the beginning, which fits with the current MW formation model. Indeed, this leaves 138 to 238 [My] for that star to form at that position. As for HD 140283, it may have started to form about $150[\mathrm{My}]$ after the beginning, which could mean that the formation of the MW began earlier than the assumed 181 [My]. However, a lot of uncertainty remains about the formation of that star very close to the sun $(0.06[\mathrm{kpc}])$. In short, the amount of unobservable mass could be quite small in the MW, meaning that rotation speeds could be largely due to cosmological gravity in play during formation, or $180[\mathrm{My}]$ after the beginning. 


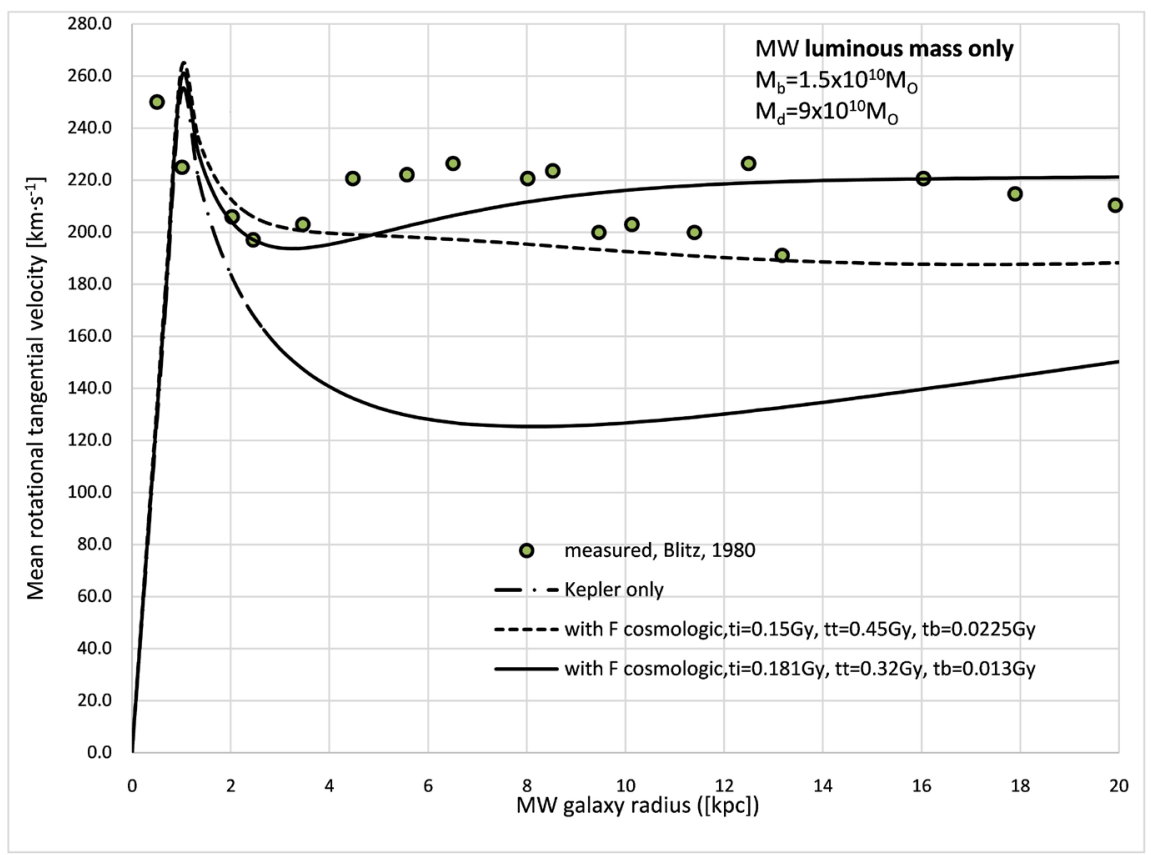

Figure 3. MW rotational velocities.

\section{M33 (SA(s)cd) (of the Triangle)}

Studying M33 to explain the radial velocity equation is an arbitrary choice, but we need a galaxy that has apparently not collided with another galaxy in the past, and which contains a large amount of dark matter. In fact, this galaxy is reportedly $85 \%$ dark matter [12]. If the dark matter is removed, the following luminous masses remain:

$$
M_{\text {Tvisibe }} \sim 0.15 M_{T} \sim 0.15\left(6 \times 10^{10} M_{\odot}\right) \sim 9 \times 10^{9} M_{\odot}
$$

To avoid making too many speculative simulations regarding center and disc masses, we chose the following values as constants:

$$
\begin{gathered}
M_{d \text { visibe }} \sim 9 \times 10^{9} M_{\odot} \\
M_{b \text { visibe }} \sim 5.0 \times 10^{8} M_{\odot}
\end{gathered}
$$

Figure 4 shows five rotation curves for M33, derived from the three remaining parameters of the equation: $t_{p} t_{T}$ and $t_{b}$, along with the rotation curve for the luminous mass only (Kepler baryonic, $F_{\Lambda}=0$ ). Note that the rotation of this galaxy is not as well represented for the greater radii. The beginning of formation is assumed to be $t_{i}=0.16$ to 0.2 [Gy]. Note also that a start time closer to the beginning increases the concave nature of the velocity profile, due to the stronger effect of the cosmological constant and the longer formation time, which flattens the velocity profile, as the cosmological constant decreases more sharply in remote areas with large radii. Further, the luminous mass is not sufficient to explain the rotation of the outer radius, as the speeds decrease sharply beyond 9 [kpc]. This confirms the presence of dark matter (baryonic non-luminous) in this galaxy because cosmological gravity alone is not enough to accurately simulate 


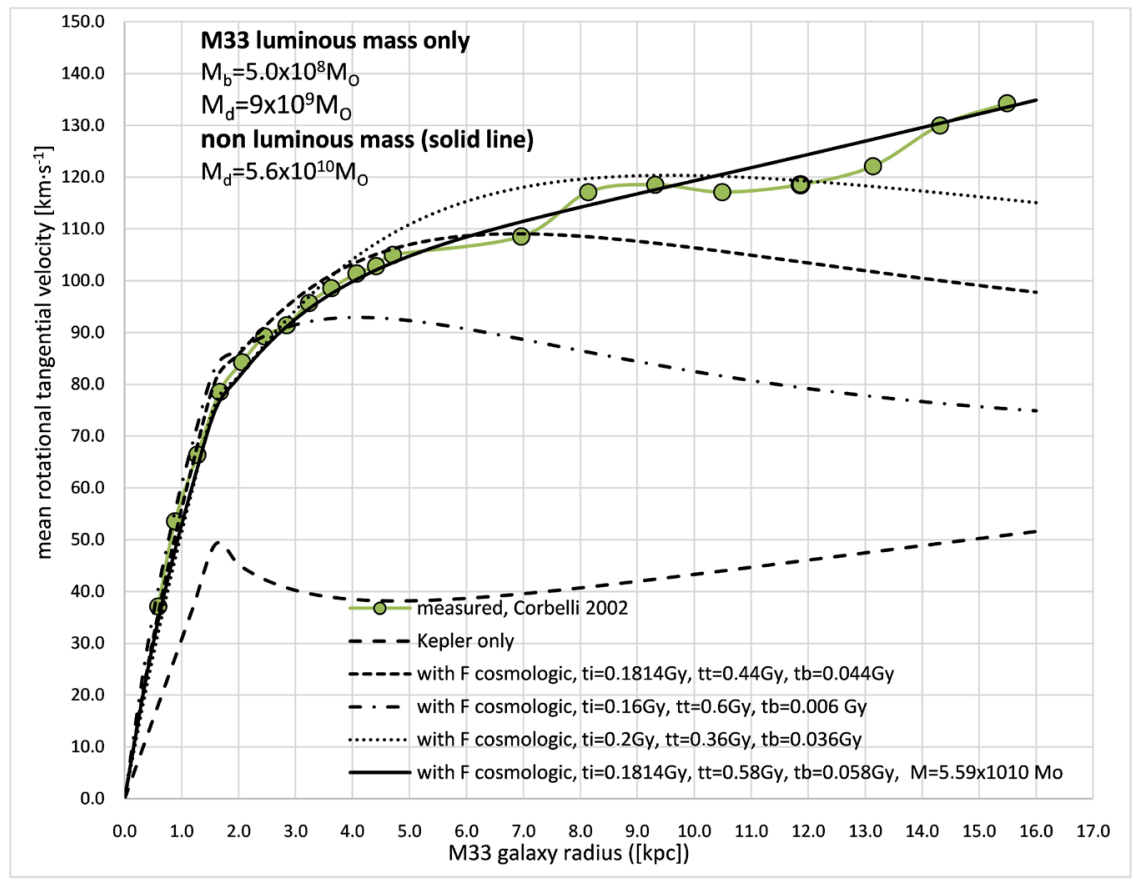

Figure 4. M33 rotational velocities.

the rotation. To determine the effects of non-luminous matter, the last curve represents a mass total that is six times greater than the estimated luminous mass $\left(5.59 \times 10^{10} M_{\odot}\right)$. Note the strong correspondence between the estimated and measured speeds, clearly showing the existence of non-uminous matter in M33 and similar galaxies.

\section{UGC12591, S0/Sa (Pegasus)}

Galaxy UGC12591 was chosen to demonstrate the significant effects of cosmological gravity, $F_{\Lambda}$, on the formation and faster rotation speeds of early galaxies. Careful studies by [13] Giovanelli et al. and [14] Xinyu Dai show very large amounts of dark matter (84\%). Removing the dark matter from the reported total mass $\left(\sim 2.7 \times 10^{12} M_{\odot}\right)$ yields the following luminous mass:

$$
M_{\text {Tbaryon }} \sim 0.17 M_{T} \sim 0.17\left(2.7 \times 10^{12} M_{\odot}\right) \sim 4.6 \times 10^{11} M_{\odot}
$$

Center mass is not specified as such. The rotation curve shows that the center mass should be greater than the disc mass to be able to closely simulate the observed rotation speeds:

$$
\begin{aligned}
& M_{\text {bvisibe }}=0.69 M_{\text {visibe }} \sim 0.69\left(4.6 \times 10^{11} M_{\odot}\right) \sim 3.18 \times 10^{11} M_{\odot} \\
& M_{d \text { visibe }}=0.31 M_{\text {visibe }} \sim 0.31\left(4.6 \times 10^{11} M_{\odot}\right) \sim 1.42 \times 10^{11} M_{\odot}
\end{aligned}
$$

Figure 5 shows two rotation curves for USG 12591, based on the three remaining parameters of the equation: $t_{p}, t_{T}$ et $t_{b}$ and the rotation curve of the luminous mass only (Kepler baryonic, $F_{\Lambda}=0$ ). The observed speed curve is relatively downward linear, indicating an early formation time. Indeed, with an adjustment of the three parameters, we see that the formation of that galaxy began 


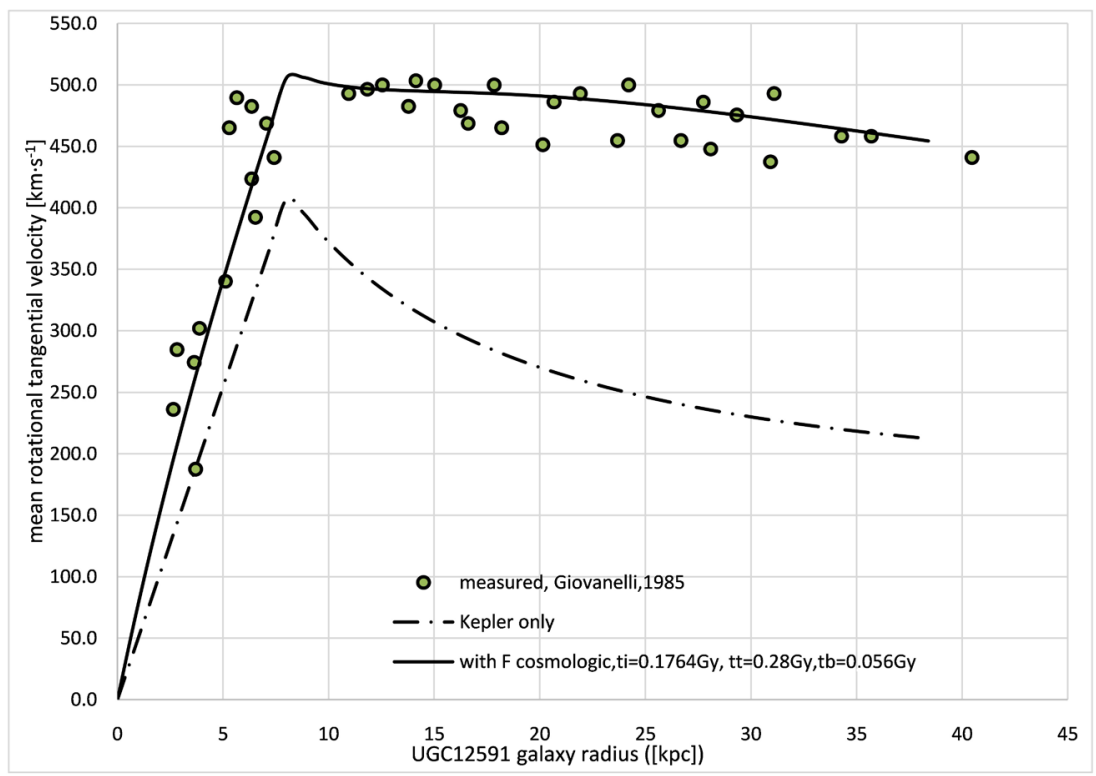

Figure 5. UGC12591 rotational velocities.

around $176[\mathrm{My}]$ and continued for about 280 [My]. Note here that this formation period is called primitive as this is when most of the mass is accumulated. Of course, the evolution of galaxies is dynamic and continuous. Finally, the rotation curve shows that this galaxy's luminous mass is sufficient to generate the observed rotation speeds. The luminous mass of this galaxy is $\sim 4.4$ times greater than that of the MW, and its center mass alone is $\sim 21$ times greater, which partly explains the great rotation speeds starting in the first $5[\mathrm{kpc}]$ of the radius.

\section{NGC3198, Sc C}

This spiral galaxy has been the object of many studies to determine its velocity profile and the mass of hydrogen gas outside its planar disc [15].

Figure 6 shows three velocity profile curves. The first shows the estimated luminous mass, $1.08 \times 10^{10} M_{\odot}$ and the three remaining parameters of the equation: $t_{p} t_{T}$ and $t_{b}$, along with cosmological gravity, $F_{\Lambda}$, calculated at the formation time of the galaxy. Note that its mass is too small to generate rotation speeds beyond 15 [kpc], even in consideration of the cosmological gravity in play at the beginning of formation, around 165 [My]. The presence of a substantial nonluminous matter halo is necessary here to explain the rotation speeds at the outer edges. For comparison purposes, the second curve shows the amount of mass added to the luminous mass to justify the rotation speeds, which is 16 times greater than the estimated mass $\left(1.78 \times 10^{11} / 1.08 \times 10^{10}\right)$. The last curve shows only the Kepler speed for the observed mass only.

This galaxy probably began to form around $181[\mathrm{My}]$ and the main accumulation may have lasted nearly 1 [Gy] (880 [My]), if the amount of lacking mass is considered. Note that non-luminous mass must be considered here, which tends to confirm that non-luminous mass can make up significant proportions of galaxies, even when cosmological gravity is in full force. 


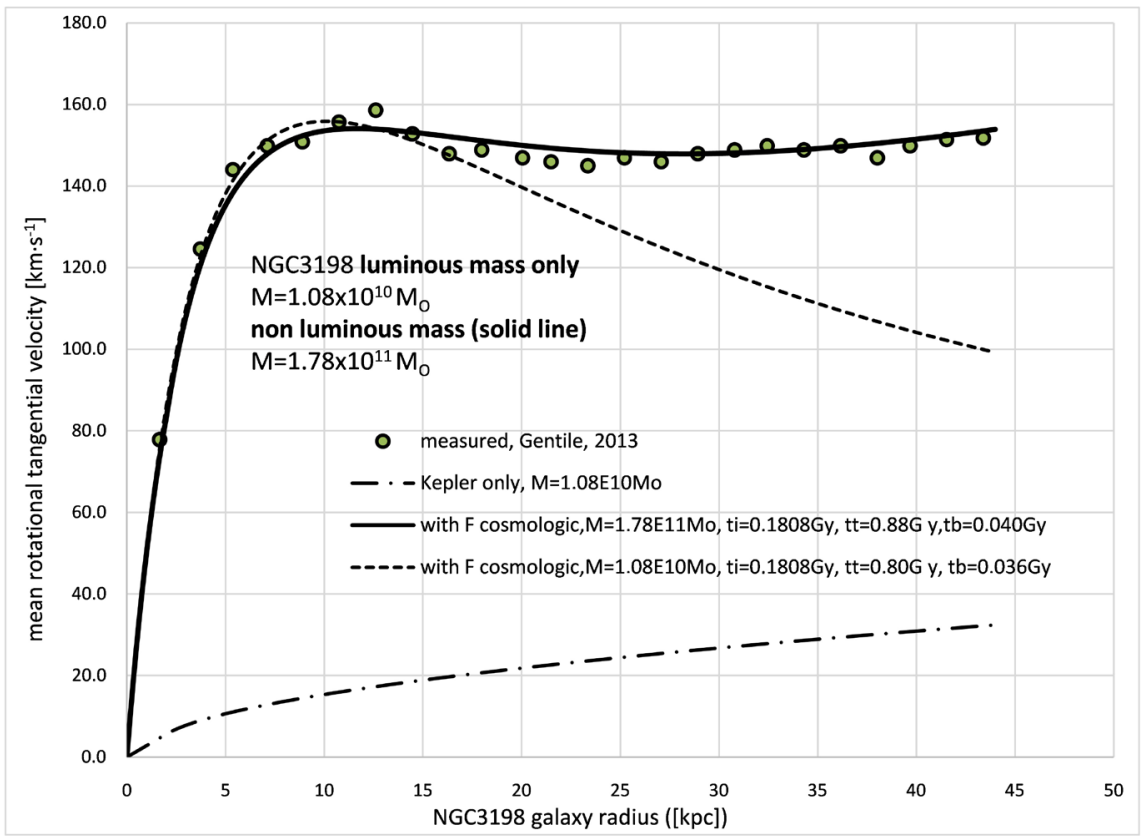

Figure 6. NGC3198 rotational velocities.

\section{UGC2885, Sc D}

One of the largest spiral galaxies observed to date has been the object of many studies. Figure 7 shows two velocity profile curves. The first for the estimated observable mass, $2 \times 10^{12} M_{\odot}$ and the three remaining parameters of the equation: $t_{p}, t_{T}$ and $t_{b}$, along with the cosmological gravity, $F_{\Lambda}$, calculated at the formation time of the galaxy. Note that the mass here is sufficient to generate the rotation speeds. The peak rotation velocity near the center is accurately predicted, but the measured peak is more spread out. The value used for the center mass, $4 \times 10^{10} M_{\odot}$, is in the same order of magnitude as the $10^{10} M_{\odot}$ estimated by Gentile [15]. Velocities at the outer radius are greater than those measured and quasi-constant at $298 \mathrm{~km} \cdot \mathrm{s}^{-1}$. However, the mass accumulation model we use is a very simple one, meaning that the galaxy's mass accumulation rate at the outer radius could be smaller, thus reducing the rate of speed increase. The last curve is the Kepler speed curve only.

The galaxy may have started forming around 180 [My] and the main accumulation probably lasted nearly 1.2 [Gy], far longer than any of the other galaxies described herein. For example, the calculated formation time for the MW is 320 [My], or 3.7 times shorter than UGC2885. The luminous mass of this galaxy is sufficient to generate its rotation speeds with the presence of the cosmological gravity.

\section{NGC253, Sculptor}

The rotation curve of this southern sky galaxy was measured by [16] Pence, with over 3700 measurements made (Fabry-Perot) along the great axis of the galaxy. Figure 8 shows an approximation of the low and high values (range) derived from the means on groups of ten values. The profile becomes nearly flat beyond 


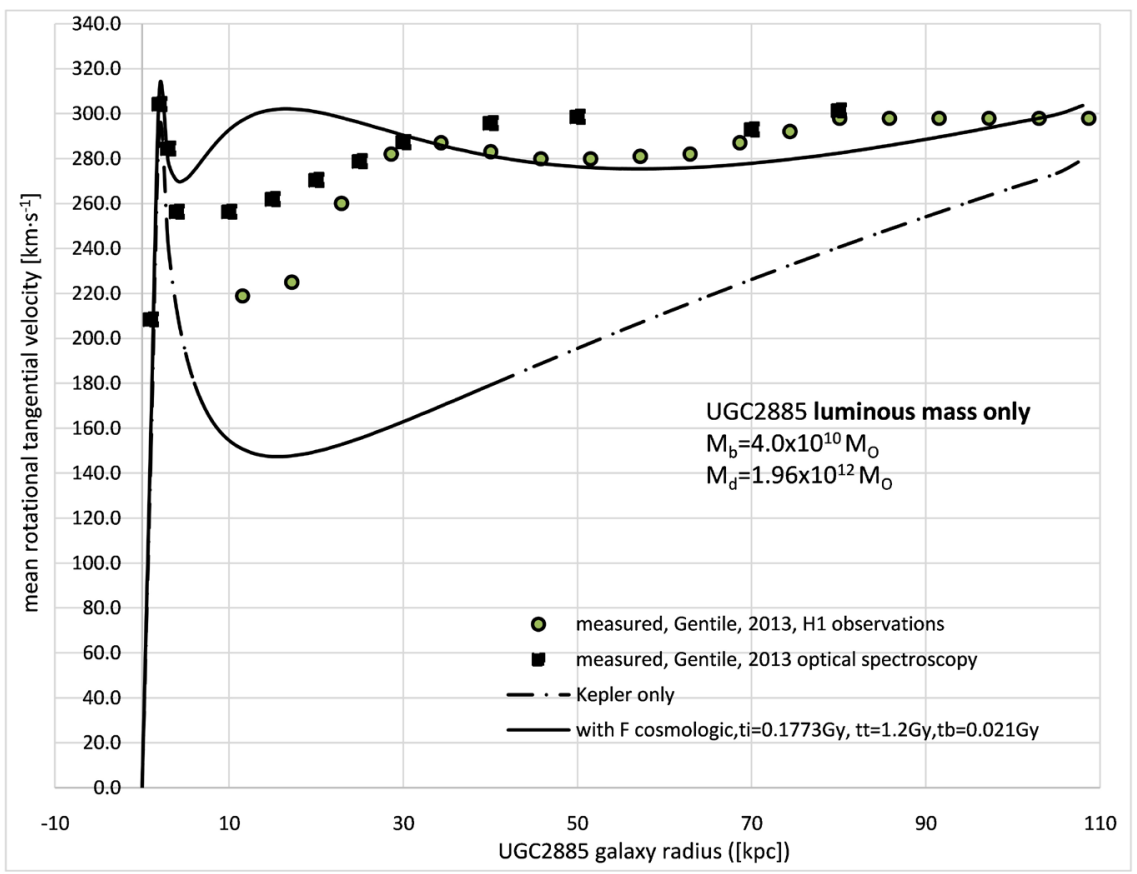

Figure 7. UGC2885 rotational velocities.

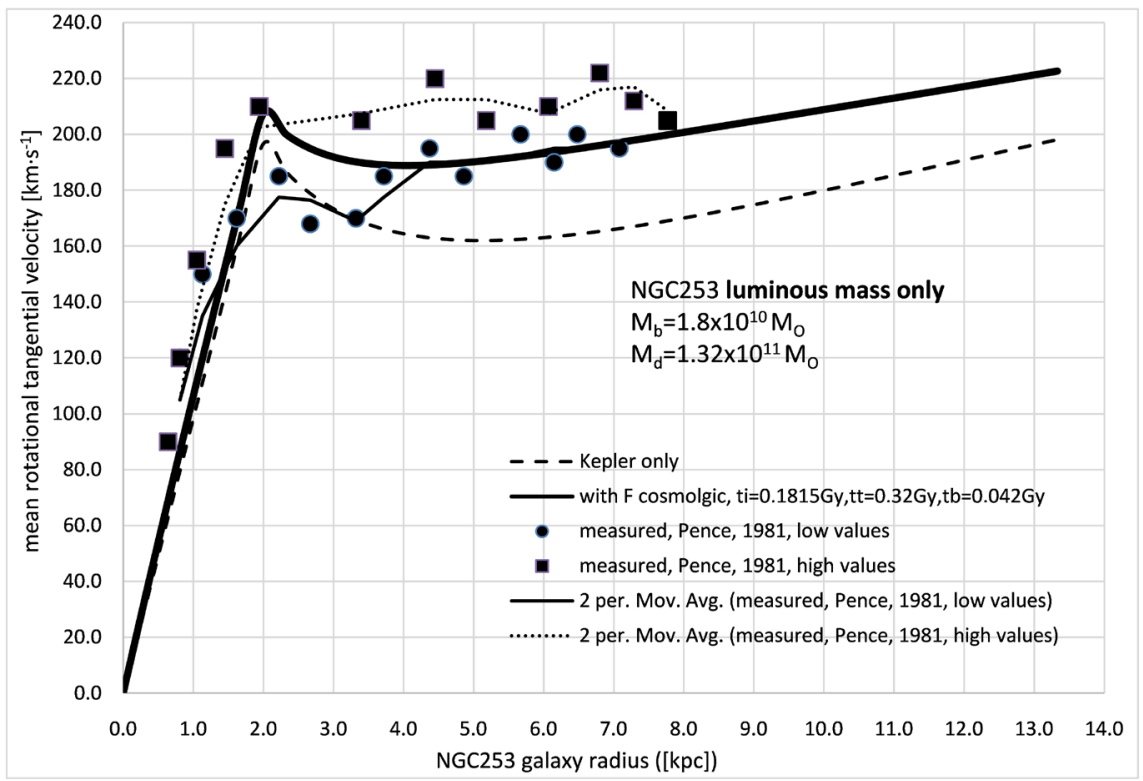

Figure 8. NGC253 rotational velocities.

an estimated $2.25[\mathrm{kpc}]$ distance from the center. In fact, Pence suggests a mean rotation speed of $205 \mathrm{~km} \cdot \mathrm{s}^{-1}$ for the measurement zone. He studied several rotation models as well as several estimated masses derived from these models (six estimated masses), varying between $1.08 \times 10^{11}$ and $1.54 \times 10^{11} M_{\odot}$, if the estimated mass of the galaxy is extrapolated beyond 7.9 [kpc], or the speed measurement zone. Indeed, the suggested rotation curve encompasses a radius of $\sim 15$ [kpc] (20.5 arc-minutes). The total mass is an estimated $1.5 \times 10^{11} M_{\odot}$, or twice that of the measurement zone (observed mass of $5-7 \times 10^{10} M_{\odot}$ for $r \leq 7.9$ 
[kpc]). Center mass is an estimated $1.8 \times 10^{10} M_{\odot}$. The velocity profile seems to correspond fairly well with measured values, with a tendency to increase. Adjustment of the velocity profile shows that this galaxy started to form before the MW, but that the center formation process took much longer to complete, or 42 [My], compared to 13 [My] for the MW. The mass of the MW center is a bit lower than NGC253, which is of larger estimated dimension, however (2.2 [kpc] compared to $1[\mathrm{kpc}]$ ), which could partly explain the longer formation time of its center. The luminous mass of this galaxy is sufficient to generate the observed rotation speeds.

\section{Irregular Dwarf Galaxy DD0161}

Dwarf galaxy DDO161 was chosen to show the later effects of cosmological gravity. [17] Côté et al. studied eight irregular dwarf galaxies with the Australian telescope, and reported large amounts of dark matter. In the case of DDO161, they predicted a large ratio of dark matter vs. luminous matter $m_{\text {dark }} / m_{\text {lumi }} \sim 8$ to 9 to explain the observed rotation speeds. The observed luminous mass (stars and gases) is:

$$
M_{\text {total }}=M_{\text {star }+ \text { gas }} \sim 6.17 \times 10^{8} M_{\odot}
$$

In this case, we assume that the galaxy's mass is only the observable kind, which increases linearly from the center to the outer radius at $\sim 6.5[\mathrm{kpc}]\left(M_{b}=\right.$ 0 ). Figure 9 shows the observed and modeled rotation curves using cosmological gravity for a formation start time of about 220 [My] and total formation time of 240 [My]. The precision of the measurements made by Côté et al. is variable, but for this example we estimate a mean uncertainty of $\sim \pm 3\left[\mathrm{~km} \cdot \mathrm{s}^{-1}\right]$. This galaxy started to form after the MW ( 40 [My] later), and formation lasted $\sim 240$ [My]. Its mean mass accumulation rate is much lower than that of the MW, or $2.57\left[M_{\odot} \cdot \mathrm{y}^{-1}\right]$ vs. $\sim 328\left[M_{\odot} \cdot \mathrm{y}^{-1}\right]$. Finally, dark matter does not have to be considered here to explain the rotation speeds of this dwarf galaxy.

\section{UDG44, Dragonfly}

To demonstrate the powerful effects of the cosmological constant, UDG44, a diffuse galaxy in the Coma cluster, was studied by [18] Van Dokkum et al., who concluded that dark matter makes up $98 \%$ of the galaxy's total mass $\left(1.9 \times 10^{10} M_{\odot}\right)$, with an observable (luminous) mass of $3.8 \times 10^{8} M_{\odot} \quad\left(r_{1 / 2}=4.6\right.$ $[\mathrm{kpc}])$. This galaxy is highly diffuse, although very massive according to researchers, and does not behave like the MW, being considered a "failed MW". Authors report that the galaxy's velocity profile is not structured, and that the mean speed is around $9\left[\mathrm{~km} \cdot \mathrm{s}^{-1}\right]$ with large dispersion (unstructured), or $\sigma \sim 47$ $\left[\mathrm{km} \cdot \mathrm{s}^{-1}\right]$. It is obvious that a Kepler rotation model would not apply here. However, to perceive the effects of cosmological gravity, the model can be used to see its effects during formation, which lasted between 1 and 5 [Gy], 3 - 15 times longer than the MW. Figure 10 shows the rotation speed relative to formation time, with a start time around 0.177 [Gy], around the same time as the MW. 


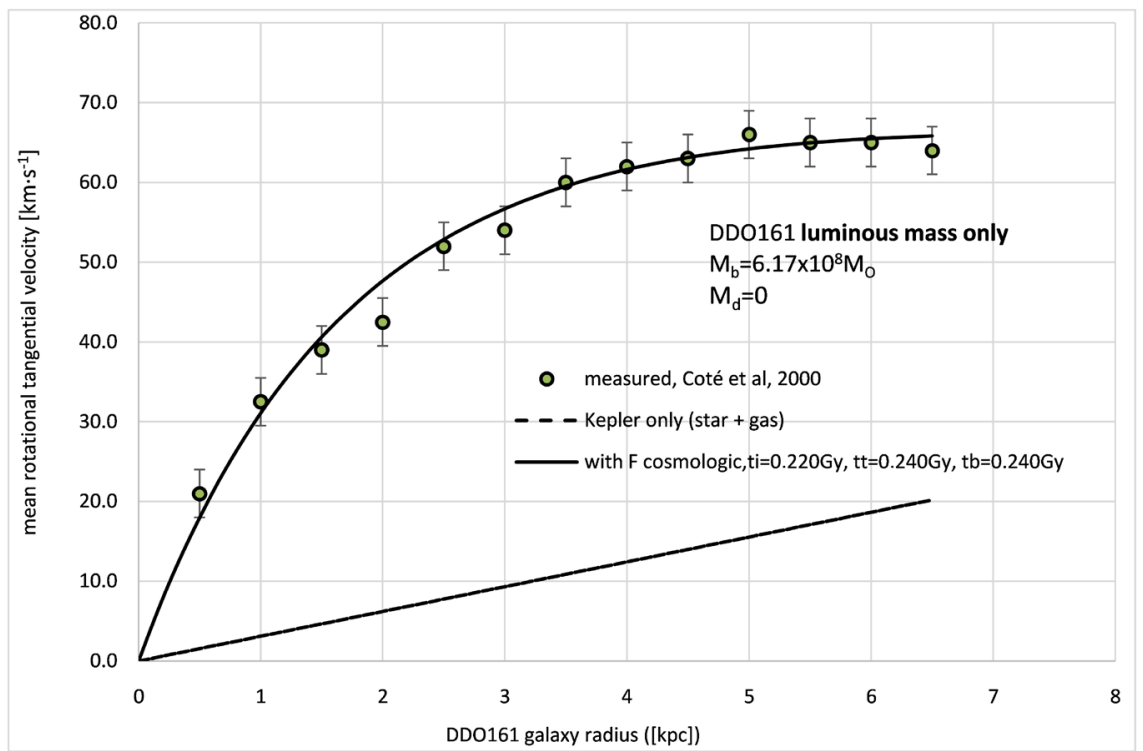

Figure 9. DDO161 rotational velocities.

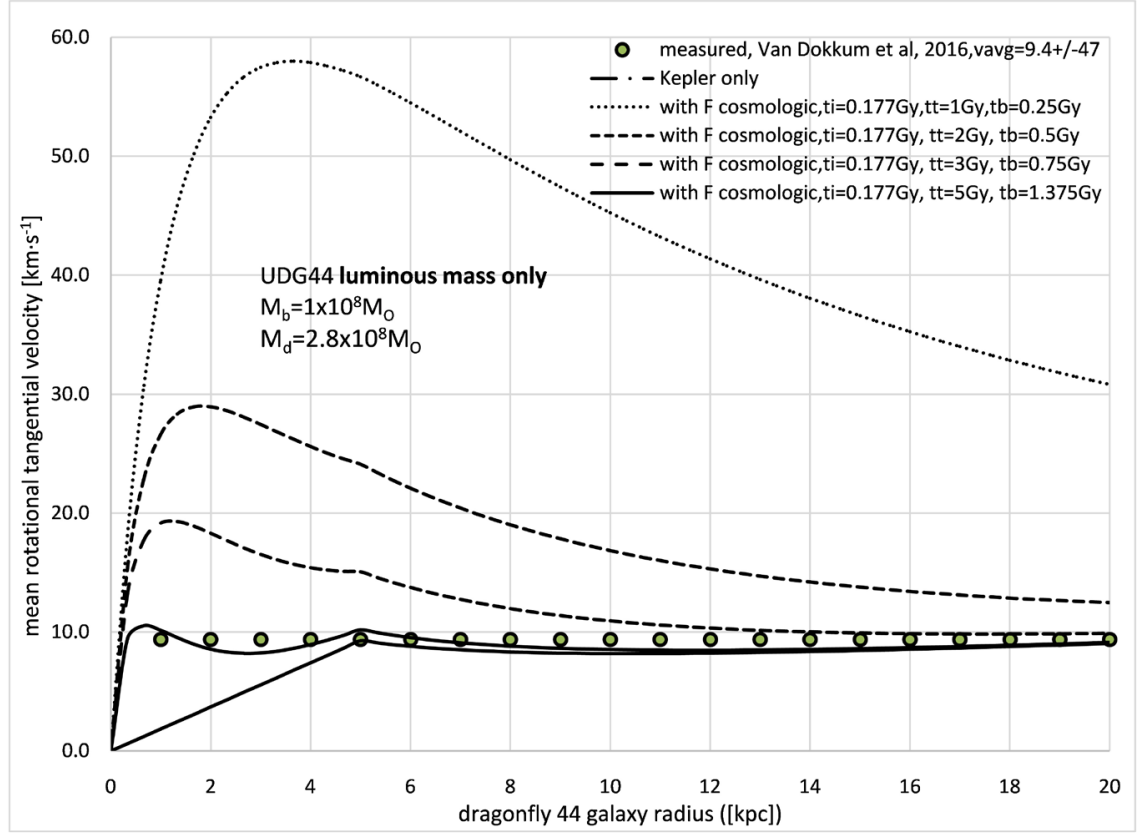

Figure 10. UDG44 rotational velocities.

Note that the longer formation takes, the greater the decrease in cosmological gravity, meaning that rotation becomes almost Kepler-type, except for speeds in the smaller radii, which do not fit well with the Kepler model $(r<5[\mathrm{kpc}])$. Note also the need to use cosmological gravity for the early formation of this galaxyas a reminder, the observations made by Van Dokkum et al. showed no established rotation. Nevertheless, we know that when a galaxy takes a very long time to form, the effects of the cosmological gravity diminish and the velocity profile points to the Kepler model; but the Kepler model is not adequate for simulating rotation speeds for smaller radii, while cosmological gravity does so relatively 
well. In short, based on this model, for some unknown reason, but likely due to a lack of neighbouring matter, this galaxy seems to have taken a very long time to form. Knowing this, with the cosmological gravity getting smaller and smaller after $\sim 1$ [Gy], the galaxy never had the impetus to generate conventional rotation, making it a diffuse galaxy or, according to this model, a late-developing galaxy from the standpoint of mass accumulation. In fact, there are very many diffuse galaxies of this type in the Coma cluster $(>40)$, which tends to confirm the idea that the matter content in this area of space is rather poor, leading to the formation of diffuse galaxies.

\section{Galaxies Cluster of Coma}

We have seen that the prediction model of the luminous mass rotation of a few galaxies, using the cosmological force of gravity, predicts fairly correctly the observed velocities. Of course, the non-luminous baryonic material exists but the quantities necessary to explain the rotational velocities are greatly diminished. Now it would be interesting to check whether on a larger scale (500 to 1000 times), this cosmological force of gravity can explain other mechanisms of rotation of matter. To do this, we apply the model of mass rotation at the scale of a galaxy cluster like that of the Coma cluster. Indeed, this cluster has been studied extensively since the 1930s with among others the studies of [19] Zwicky and thereafter those of [20] Mayall, [21] Van Albada, [22] Omer et al., [23] Pebbles, [24] Rood et al., [25] Kent et al., [26] Merrit, [27] White et al. and recently [28] Gavazzi. In summary, the various studies have all shown, to varying degrees, that the observed velocities of the $\sim 1000$ galaxies of the cluster can not be explained again by the presence of the luminous mass only estimated from the brightness-mass of galaxies ratio $\left(M_{\odot} / L_{\odot}\right)$. It was also with the study of this cluster that the concept of dark matter was proposed (Zwicki). The application of the rotation model is more complicated in the case of a galaxy cluster for mainly four reasons are:

- The clusters are much larger than the galaxies, with a rather spherical shape as well as a difficult boundary to determine precisely considering the surrounding objects.

- The clusters are remote and the Hubble-Lemaitre expansion effect is considerable (Hubble-Lemaitre flow).

- Most galaxies and other objects in the cluster are not bright and more difficult to characterize.

- The velocities of the galaxies are observed from a line of sight that crosses the cluster in the direction of sight which causes a large variation of the observed velocities.

For the Coma cluster, for predicting the velocity of galaxies, we need to estimate the luminous mass $M$, the time of the start of formation of the cluster $t_{p}$ the formation time of the cluster $t_{T}$ and the size of the cluster $r$. As an example, White et al., estimates the mass of stars and hot gas in the cluster from the observations of [29] to: 


$$
\begin{gathered}
M_{\text {star }}=1 \pm 0.2 \times 10^{13} h_{70}^{-1} M_{\odot} \sim 1.43 \pm 0.3 \times 10^{13} M_{\odot} \\
M_{\text {gas }}=5.45 \pm 0.98 \times 10^{13} h_{70}^{-5 / 2} M_{\odot} \sim 13.3 \pm 2.4 \times 10^{13} M_{\odot} \\
M_{\text {star }+ \text { gas }}=1.47 \pm 0.27 \times 10^{14} M_{\odot}
\end{gathered}
$$

White estimates that the missing mass in the Coma cluster is about $90 \%$ of the total or $\sim 9$ times the luminous Mass. The following Table 1 shows some values of the characteristics of the Coma cluster according to several authors and different databases.

We see that the observed and estimated characteristics are relatively variable between the authors. However, one observation is constant, i.e. the estimated observed (luminous) mass is less than 2 to 10 times compared to that estimated necessary to explain the observed rotational velocities of cluster galaxies. For the application of the model, several choices of values are possible. We arbitrarily select the values of Kent et al. [25] which present a review of the authors' data before them and the luminous mass estimated by White et al., i.e.:

\begin{tabular}{|c|c|c|c|c|c|}
\hline reference & $\begin{array}{c}\text { radius } \\
{\left[{ }^{\circ}\right]}\end{array}$ & $\begin{array}{c}\text { radius, } \boldsymbol{r} \text { (with } d_{\odot} \sim 103 \mathrm{Mpc}, \\
H=73 \mathrm{~km} \cdot \mathrm{s}^{-1} \cdot \mathrm{Mpc}^{-1} \\
\text { Nasa) }[\mathrm{Mpc}]\end{array}$ & $\begin{array}{c}\text { Mean radial velocity, } \\
\left\langle\bar{V}_{R}\right\rangle\left[\mathrm{km} \cdot \mathrm{s}^{-1}\right] \\
{[\text { N galaxies }]}\end{array}$ & $\begin{array}{l}\text { Luminous Mass } \\
\qquad M_{I}\left[M_{\odot}\right]\end{array}$ & $\begin{array}{l}\text { Total Mass } M_{T} \\
\text { (various models } \\
\text { and } H \text { ) }\left[M_{\odot}\right]\end{array}$ \\
\hline Zwicky [19] & $\sim 2^{\circ} .25$ & $\begin{array}{l}\sim 13.8\left[H=55 \mathrm{~km} \cdot \mathrm{s}^{-1} \cdot \mathrm{Mpc}^{-1}\right] \\
\sim 10.4\left[H=73 \mathrm{~km} \cdot \mathrm{s}^{-1} \cdot \mathrm{Mpc}^{-1}\right]\end{array}$ & & - & $4.5 \times 10^{13}$ \\
\hline Mayall [20] & $\sim 3^{\circ} .3$ & $\sim 5.9$ & $\begin{array}{c}\sim 6920 \\
47\end{array}$ & - & - \\
\hline Van Albada [21] & $\sim 2^{\circ}$ & $\sim 3.6$ & - & - & - \\
\hline [30] Abell (1965) & $\sim 2^{\circ} .5$ & $\sim 4.5$ & - & $6 \times 10^{12}$ & $2-5 \times 10^{14}$ \\
\hline Omer Jr, Page et al. [22] & $\sim 1^{\circ} .65$ & $\sim 3$ & - & - & - \\
\hline Peebles [23] & $\sim 2^{\circ} .5$ & $\sim 4.5$ & $\begin{array}{c}\sim 6925 \\
42\end{array}$ & - & $\begin{array}{c}\left(1.46 \times 10^{15} h_{50}^{-1} \mathrm{e}^{ \pm 0.3}\right) \\
2.1-3.9 \times 10^{15}\end{array}$ \\
\hline Rood, Page et al. [24] & $\sim 3^{\circ} .7$ & $\sim 6.7$ & $\begin{array}{l}\sim 6888 \\
102\end{array}$ & $\begin{array}{c}\left(\sim M_{t} / 7\right) \\
3.88-5.92 \times 10^{14}\end{array}$ & $2.72-4.15 \times 10^{15}$ \\
\hline Chincarini and Rood [32] & $\sim 5^{\circ}$ & $\sim 9$ & $\begin{array}{c}0^{\circ}-1^{\circ} .67 . \sim 6946 \\
1^{\circ} .67-3^{\circ} . \sim 7059 \\
3^{\circ}-5^{\circ} . \sim 6909\end{array}$ & - & - \\
\hline [31] Abell & - & $\begin{array}{c}\sim 2.144 \times 10^{23} 8[\mathrm{~m}] \\
\sim 6.95\end{array}$ & $\begin{array}{l}\sim 6888 \\
\text { Rood }\end{array}$ & $\begin{array}{l}\left(\sim M_{t} / 3-M_{t} / 2\right) \\
1.6-10 \times 10^{14}\end{array}$ & $5-30 \times 10^{14}$ \\
\hline Kent and Gunn [25] & $\begin{array}{l}\sim 4^{\circ} .7 \text { to } \\
6^{\circ} .8\end{array}$ & $\sim 8.5$ to 12.2 & - & - & $\begin{array}{c}\left(2.9 \times 10^{15} h_{50}^{-1}\right) \\
5.8 \times 10^{15}\end{array}$ \\
\hline Merritt [26] & $\begin{array}{c}\sim 13^{\circ} \\
\text { (model) }\end{array}$ & $\sim 23$ & $\begin{array}{c}\sim 6932 \\
296\end{array}$ & $\begin{array}{c}\left(\sim M_{t} / 3\right) \\
5.3-6.3 \times 10^{14}\end{array}$ & $\begin{array}{c}\left(1.6-1.9 \times 10^{15} h_{100}^{-1}\right) \\
1.6-1.9 \times 10^{15}\end{array}$ \\
\hline White, Navarro et al. [27] & - & 6.95 (Abell. 1977) & - & $1.47 \pm 0.3 \times 10^{14}$ & $1.21 \pm 0.26 \times 10^{15}$ \\
\hline Gavazzi, Adami et al. [28] & - & - & - & - & $\begin{array}{l}\left(5.1_{-2,1}^{+4,3} \times 10^{14} h_{70}^{-1}\right) \\
0.42-1.34 \times 10^{15}\end{array}$ \\
\hline
\end{tabular}

$$
r_{T} \sim 10.35[\mathrm{Mpc}]
$$

Table 1. Several authors of Coma cluster studies. 


$$
M_{l} \sim 1.5 \times 10^{14} M_{\odot}
$$

The following graphs show the results obtained for the Coma cluster. In order to allow for longer development and variable accretion in time of mass of a cluster compared to a galaxy, the expression of the growth of the radius $r$ (and mass) is modified slightly like this:

$$
r(t)=\alpha t^{b}=\frac{t^{b}}{t_{T}^{b}} r_{T}
$$

With $0<b<1$

For a circular rotation model, the tangential velocity is expressed as:

$$
v_{t}^{2}=G\left[\frac{\alpha^{2} t^{2 b}}{r_{b}^{3}} M_{b}+\frac{\alpha^{2} t^{2 b}-r_{b}^{2}}{\alpha t^{b}\left(r_{T}^{2}-r_{b}^{2}\right)} M_{d}\right]+\frac{k_{\Lambda} c^{2} \alpha^{2} t^{2 b}}{6\left(t_{i}+t\right)^{4}}
$$

We know that for spherical geometry, mean quadratic radial velocity (line of sight) can be expressed from the quadratic velocity $v$, i.e.:

$$
\left\langle\bar{V}_{R}\right\rangle^{2}=3 v^{2}=3 v_{t}^{2}
$$

Three unknowns are to be determined, the time of the start of the formation of the cluster $t_{i}$, the duration of the formation of the cluster $t_{T}$ and the variable speed of progression of the formation of the cluster with exponant $b$. Several combinations are possible but we possess the measured values of the radial velocity of the galaxies according to the radius $\left\langle\bar{V}_{R}\right\rangle$ of the cluster as well as the standard deviations of the velocities $\sigma_{R}$. The following Figures 11-13 present the profile of the standard deviation $\sigma_{R}$ for different plausible combinations of $t_{p} t_{T}$ and (b) and the measures taken by Kent [25], Rood [24] and [32] Chincarini. Several observations can be made:

- The standard deviations $\sigma_{R}$ of $\left\langle\bar{V}_{R}\right\rangle$ are variable for small radii, calibration is more difficult for this area.

- The high values of $t_{T}(>3-4$ [Gy]) do not fit well with the values found for $r>$ 4000 [kpc].

- The lower values of $t_{i}(0.6[\mathrm{~Gy}])$ do not fit well with the values found.

- The lower values of $b(<0.5[\mathrm{~Gy}])$ do not correspond well to the values found. In summary, we observe that the beginning of the formation of the cluster is posterior to $\sim 0.6$ [Gy] and the duration of the formation is less than $\sim 2.5$ [Gy]. In addition, the growth rate of the cluster appears to be higher at the beginning $(b<1)$. If we choose the following preferred values $\left(t_{i}=0.7\right.$ [Gy], $t_{T}=2.2$ [Gy] and $b=0.5)$, we obtain the following velocity curves for the Coma cluster (Figure 14).

We can draw the following conclusions about the Coma cluster and the cosmological force $F_{\Lambda}$.

1) Excluding this cosmological force, it is not possible to reproduce the observed radial velocities using only the luminous mass and gravitational force only. Indeed, the rotational velocities are too low. If the mass of the cluster is increased by 40 times, the observed elevated velocities can be obtained without the cosmological gravitational force. 


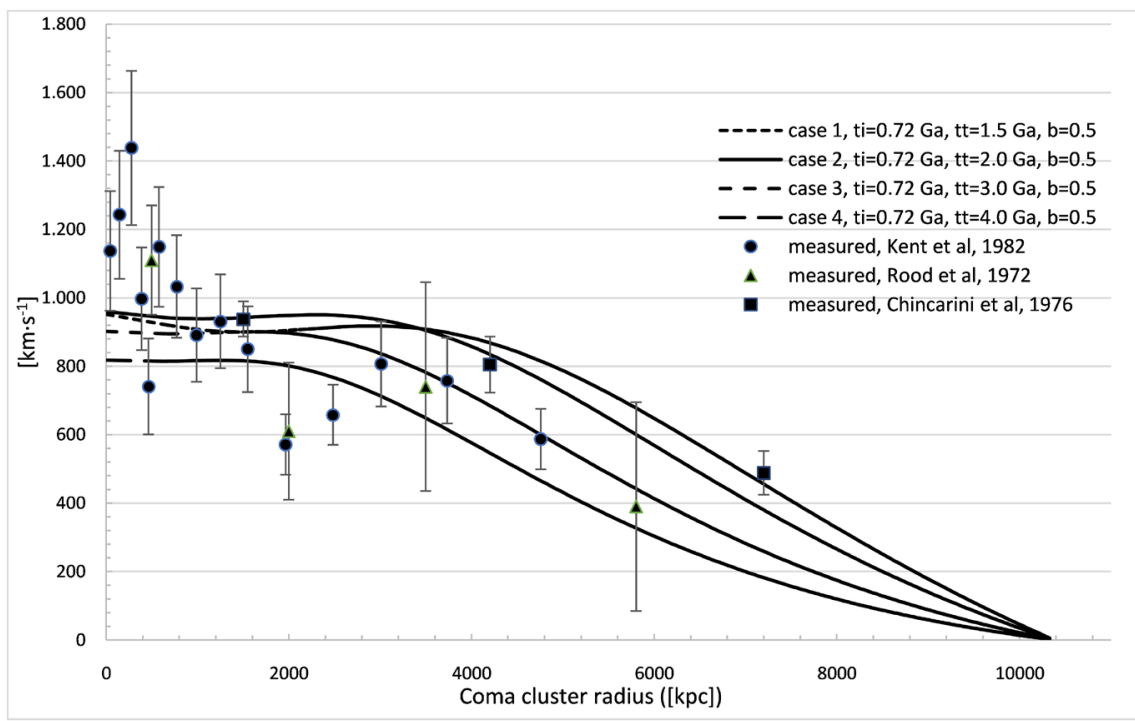

Figure 11. Coma cluster radial velocity dispersion profil.

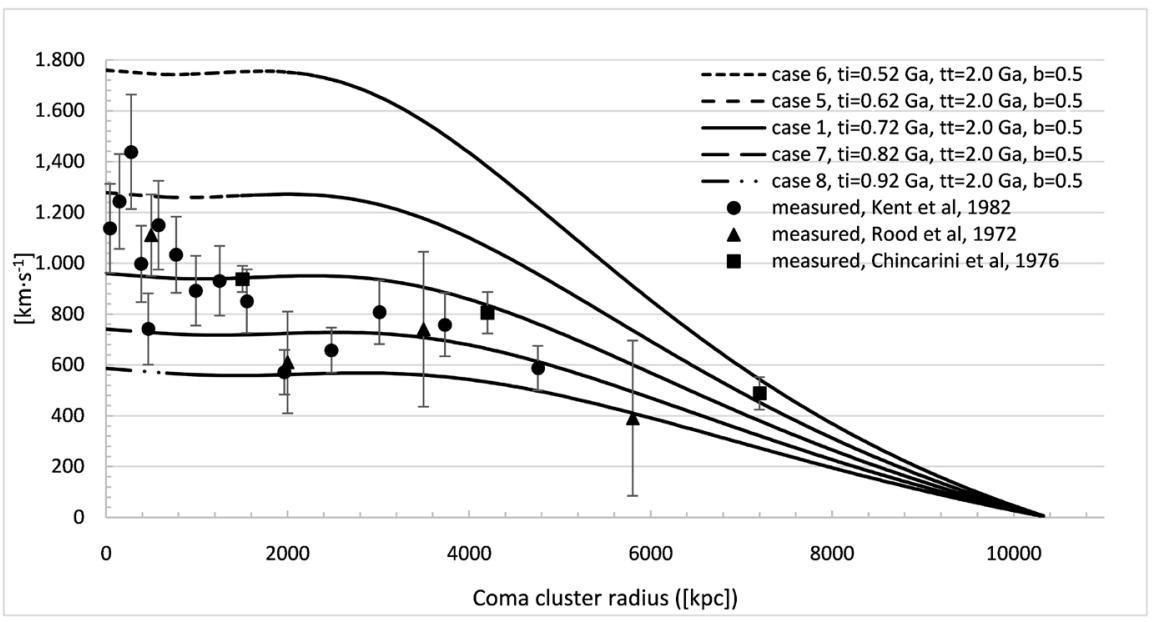

Figure 12. Coma cluster radial velocity dispersion profil.

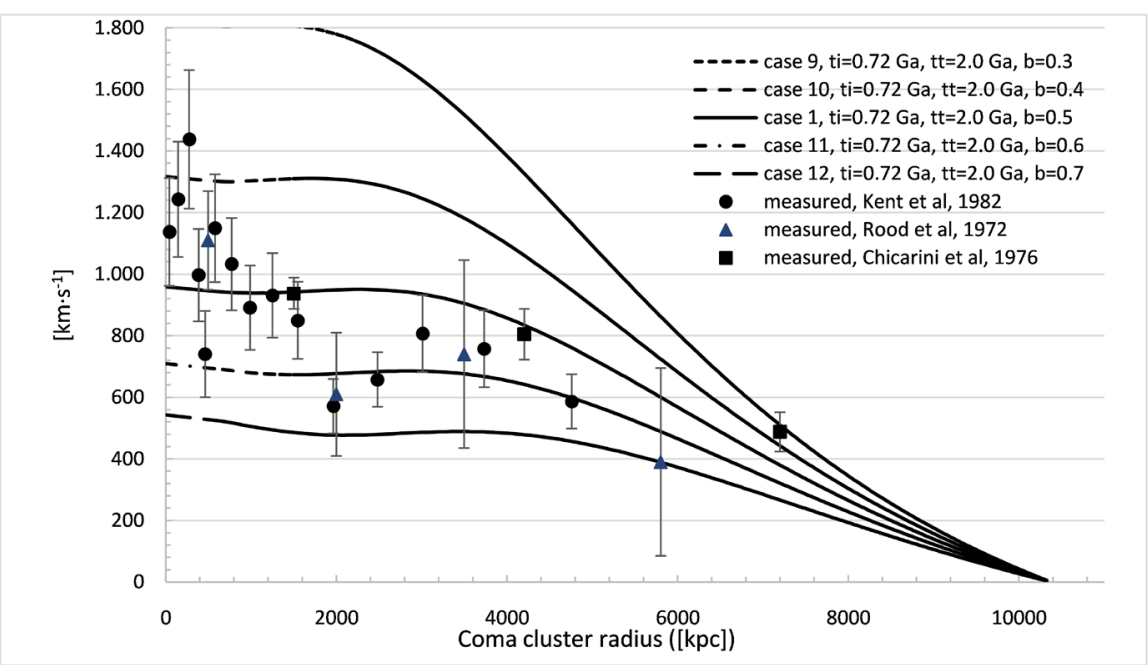

Figure 13. Coma cluster radial velocity dispersion profil. 


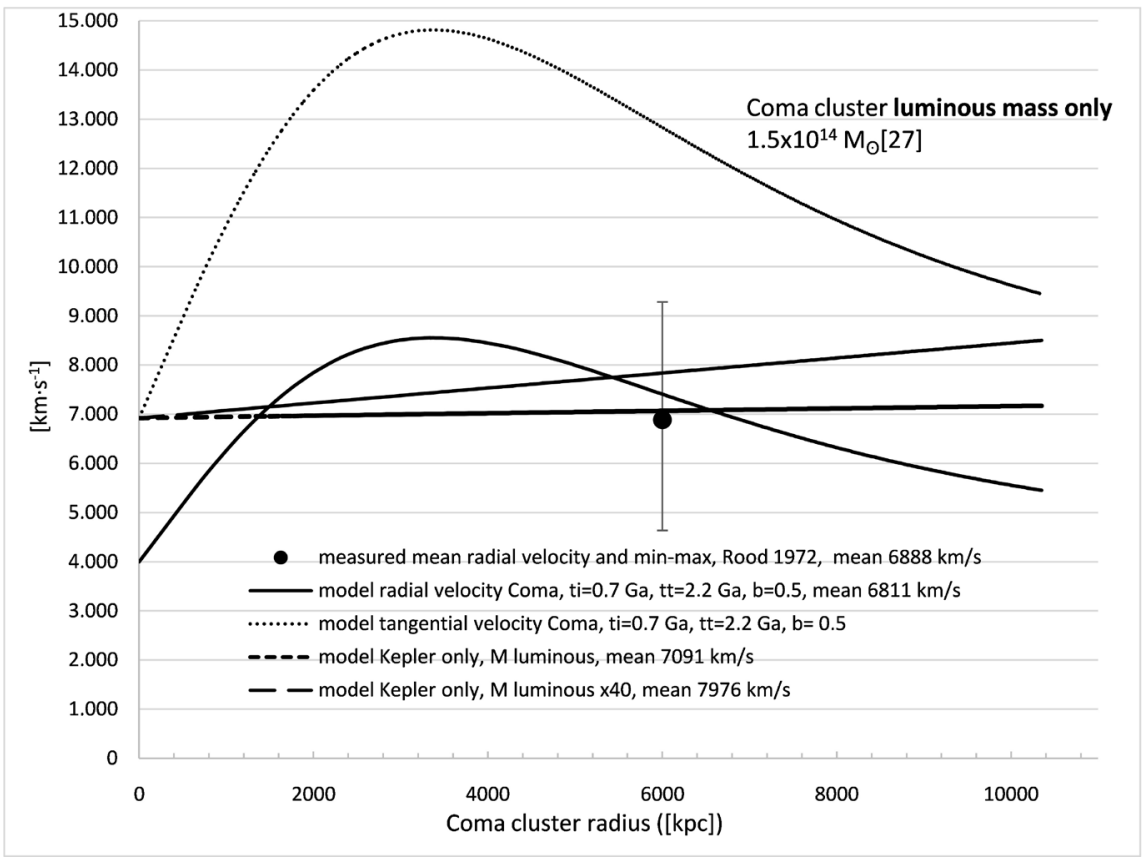

Figure 14. Coma cluster radial velocity dispersion profil.

2) The cosmological constant $\Lambda$ is to much decrease for a time of formation initial $t_{i}$ of 0.7 [Gy] compared to that of the MW of 0.18 [Gy] (228 times smaller) but, the $r$ dimension of the Coma cluster is $\sim 500$ times greater which allows to maintain the cosmological gravitational force in the case of a cluster of galaxies.

3) As an approximate comparison, the beginning of the formation of the Coma cluster is located $\sim 240$ [My] later than the formation of a dwarf galaxy like DDO161 but $\sim 670$ [My] before the end of the formation of a giant galaxy such UGC2885. This suggests that the beginnings of the formation of clusters, similar to that of Coma, began when galaxies like the MW were already present but during the formation of the cluster which has duration $\sim 2.2$ [Gy], the larger galaxies continued to develop and they have reached the maturity of their development before the end of the formation of the cluster.

4) If we look at Kent's [25] database, we can find radial velocity values $\left\langle\bar{V}_{R}\right\rangle$ as high as $13,000-17,000\left[\mathrm{~km} \cdot \mathrm{s}^{-1}\right]$. This confirms that the values of the tangential velocity curve $v_{t}$. In Figure 15 are realistic because the maximum observable speeds of $\left\langle\bar{V}_{R}\right\rangle$ are precisely those of this tangential velocity when this one is practically aligned with the line of sight. However, without the cosmological gravitational force (Kepler only), it is not possible to obtain such large values of $\left\langle\bar{V}_{R}\right\rangle$ unless you increase the mass of the cluster by a $300 \mathrm{x}$ factor.

5) It is possible to estimate the evolution time of the UDG dragonfly 44 (Coma) compared to the time of formation of the Coma cluster. If we consider the angular separation of dragonfly 44 with the estimated center of the cluster, we find $\sim 1.035^{\circ}$ (Nasa Heasarc). This angular distance converted in length gives $\sim 1.86$ [Mpc]. This gives a time of evolution of dragonfly $44 \sim 2.2(1.86 / 10.35)$ [Gy] or $\sim 0.395$ [Gy] after the beginning of the cluster $(0.7$ [Gy]) or a time of evolution 


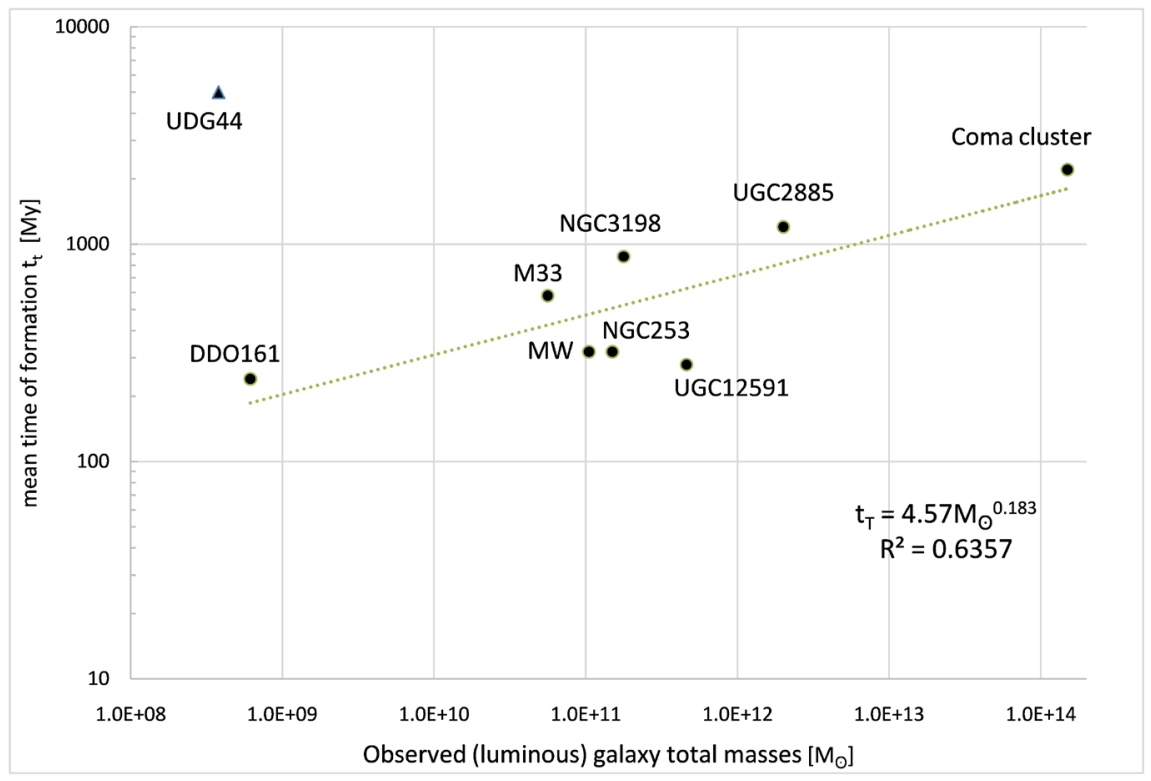

Figure 15. Galaxies tendency of main formation time fonction of luminous masses.

of dragonfly 44 of $(1.09-0.177$ [Gy]) $\sim 0.91$ [Gy] when joined to the cluster which is a lower value than the formation time of dragonfly 44 estimated at $\sim 5$ [Gy]. This seems to indicate that the formation of galaxies is parallel to that of galaxy clusters, that is, the UDG galaxies as dragonfly 44 are probably not fully mature or formed when joined to the formation of a galaxy cluster.

In summary, with respect to the formation of a cluster of galaxies using the cosmological gravitational force, we observe that as in the case of galaxies, the formation appears faster than most estimates. However, lately, [33] Wang et al. observed a cluster called (protocluster) consisting of 17 massive galaxies and $\mathrm{x}$-ray emission observations, suggests that the cluster formed rapidly from a dense nucleus of $80[\mathrm{kpc}]$ like a galaxy or even a black hole and the universe had only 2.5 [Gy]. This suggests that the rapid formation of the clusters does indeed exist. Besides other clusters or protocluster seems to have been discovered according to the same authors.

\section{Summary of the Galaxy Rotation Model}

We have seen that the five-parameter model performs relatively well for the simulation of mass velocity profiles for the seven galaxies and Coma cluster described above. The model shows mainly early formation of galaxies, which is not usually considered, although recent observations have shown that organized strucutures did exist as early as 400 [My]. [34] Wang et al. have observed the existence of 39 massive and mature galaxies only 2 [Gy] after the beginnings of the universe. Lately, the ALPINE-ALMA project, Le Fèvre et al. [10], reports the existence of 118 galaxies already formed from 1 to 1.5 [Gy] after the origin. However, the mass accumulation model (radius growth) is very basic, and a full-capacity accumulation model based on existing forces would be more realistic and would surely yield more accurate galactic growth rates. The model uses 
cosmological gravity, a major force during the initial billion years when the first galaxies were formed. Also, variation of the constant $G$ (as $G(r, t))$, used to adjust gravity forces for large radii, is not used in this mass rotation speed simulation model [35].

Observation of early galaxies is difficult due to their low brightness. [36] Bouwens et al. reported that very few galaxies were formed before 700 [My]. However, some very old stars have been detected in the MW, which tends to confirm primitive formation of the galaxy before 700 [My]. For example, GN$\mathrm{Z} 11$ is located $400[\mathrm{My}]$ after the beginning. If a fast formation time period is assumed, say 200 [My], this brings the start of formation to about 200 [My] after the beginning, which is in the order of magnitude of $t_{i}$ values yielded by this model. Galactogenesis is still a very wide-open question and delaying the start times of galaxy formation can be done by changing the accumulation rates or increasing the age of the universe, because cosmological gravity depends on the cosmological constant, which is cosmic time dependent, $t_{\Omega}$. With cosmological gravity in play, there is less of a need to turn to lacking or unobserved mass (like dark matter-existing but non-luminous baryonic matter) to explain the rotation patterns of many galaxies. In brief, from the standpoint of observable and unobservable masses of these eight galaxies, we had recourse to dark matter (non-luminous) for two of the galaxies (M33 and NGC3198). As for the other galaxies, only the observable luminous mass and cosmological gravity were used.

What is interesting with this model is the fact that it was derived from the analysis of a model of the universe that estimates the evolution of energy to calculate new dynamic parameters, such as the cosmological constant and cosmological gravity. Moreover, the mass rotation model for galaxies provides estimated formation times from early formation, $t_{p}$ as well as formation time, $t_{p}$ by adjusting these two parameters with the observed rotation curves and observed masses. In our opinion, no other model uses these dynamic parameters for the formation of galaxies with the cosmological constant. Finally, the mass accumulation model described herein is a very simple one; nevertheless, the results of rotation speed simulations are promising. Of course, a much greater number of galaxies should be studied with this rotation model to further improve and develop its potential. However, we can generate a graph (Figure 15) showing the relation between total observed mass and formation times of these galaxies, with the exception of UDG44, which does not fit the model due to a significant amount of time of formation. The graph shows that formation time increases with total mass, which is quite plausible.

\section{Relative Position of Galaxies}

While this may seem unlikely, using the matter expansion equation and estimated initial time of formation of a galaxy center $\left(t_{i}\right)$, we can derive an approximate cosmic time location of that galaxy relative to the MW. For example, using the estimated times to adjust rotation, we found that the formation of UGC12591 
galactic center was completed around $176[\mathrm{My}]$ after the beginning (see Table 2 below). The estimated distance of this galaxy is $\sim 394 \pm 133$ [My], or a maximum \pm 527 [My], which corresponds with an age limit for the formation of the center in the range of 13.27 [Gy] $t \leq 14.33$ [Gy]. With a scale factor of an initial sphere of $1[\mathrm{~Gy}]\left(t_{\Omega} / t=76.1[\mathrm{~Gy}] / 1[\mathrm{~Gy}]\right)$, we find that the center of this galaxy was probably formed between 174 [My] and 188 [My] (13.27 [Gy] /76.1 [Gy] and $14.33[\mathrm{~Gy}] / 76.1[\mathrm{~Gy}]$ ) after the beginning. Comparing the above values with the rotation curve (176 [My]), we find that this galaxy started to form around 5 [My] before the MW. Of course, these data are the result of manual adjustments of parameters $m_{b}, m_{d}, t_{p}, t_{b}$ et $t_{T}$, which leaves a fairly large margin of error. However, if the notion of this model for an approximate time of formation of the bulbe of these galaxies is accepted and we also accept that there is a characteristic or preferable time to the formation of galaxies, this could open a way to determine a preferred direction towards the beginning. This idea of a definite direction was addressed by [37] Zhou et al. Indeed, from the study of observed acceleration variations, $g_{o b s}$, they determined two precise but diametrically opposed galactic directions $(1, b)$ and $\left(1+180^{\circ},-b\right)$, where the accelerations of 147 galaxies show systematic differences that lead to two most likely directions. They used the MOND theory to derive these directions, along with values for $a_{0}$ which, as we will see later, are fundamentally related to the cosmological constant, which depends on cosmic time, and to the formation time of the structure. Therefore, a more methodical study of the rotation of many galaxies around the galactic sphere would help to determine, with rotation curves and estimated masses, if a formation trend before or after the MW could yield a specific direction, and thus confirm or reject the notion of a possible direction towards the beginning.

\section{MOND Theory and Cosmological Constant}

The cosmological constant can be used to find a possible fundamental explanation for the MOND theory. Indeed, by equalizing the expression of rotation speed for the mass of a great structure, as predicted with the MOND theory, to that obtained using conventional and cosmological gravity, we get the following equation of equality:

$$
\frac{v^{2}}{r}=a_{\mathrm{MOND}}=a_{G+\Lambda}
$$

Or,

$$
v_{\mathrm{MOND}}=\sqrt[4]{G M a_{0}}=\sqrt[2]{\frac{G M}{r}+\frac{\Lambda c^{2} r^{2}}{6}}
$$

From this expression, a more fundamental expression is obtained for the constant $a_{0}$ of this theory $\left(a_{0} \sim 1.2 \times 10^{-10}\left[\mathrm{~m} \cdot \mathrm{s}^{-2}\right]\right)$, after a few manipulations, we get:

$$
a_{0}=\frac{G M}{r^{2}}+\frac{\Lambda c^{2} r}{3}+\frac{\Lambda^{2} c^{4} r^{4}}{36 G M}
$$


Table 2. Beginning of formation time of some galaxies.

\begin{tabular}{|c|c|c|c|c|c|c|}
\hline \multicolumn{4}{|c|}{ FROM OBSERVATION } & \multicolumn{3}{|c|}{ FROM ROTATION CURVE } \\
\hline Galaxy & $\begin{array}{c}d_{\odot} \\
\text { (now) } \\
{[\mathrm{My}]}\end{array}$ & $\begin{array}{c}t / t_{\Omega} \\
(\min \text { and } \max ) \\
{[-]}\end{array}$ & $\begin{array}{c}t_{i} \\
(\min \text { and } \max ) \\
{[\mathrm{Gy}]}\end{array}$ & $\begin{array}{c}\boldsymbol{t}_{\boldsymbol{i}} \\
{[\mathrm{Gy}]}\end{array}$ & $\begin{array}{c}t_{b} \\
{[G y]}\end{array}$ & $\begin{array}{c}\left(t_{i}\right)^{m w}-t_{i} \\
{[\mathrm{My}]}\end{array}$ \\
\hline MW & 0 & $13.8 / 76.1$ & 0.1814 & 0.1814 & 0.013 & 0 \\
\hline UGC12591 & $394 \pm 133$ & $13.27 / 76.1-14.33 / 76.1$ & $0.1744-0.1882$ & 0.1764 & 0.056 & $\sim 5.0$ \\
\hline UGC2885 & 310 & $13.49 / 76.1-14.11 / 76.1$ & $0.1772-0.1854$ & 0.1773 & 0.021 & $\sim 4.1$ \\
\hline NGC3198 & 47 & $13.75 / 76.1-13.85 / 76.1$ & $0.1807-0.1819$ & 0.1808 & 0.039 & $\sim 0.61$ \\
\hline M33 & 2.38 à 3.07 & $13.797 / 76.1-13.803 / 76.1$ & $0.1813-0.1814$ & 0.1814 & 0.058 & $\sim 0.03$ \\
\hline UDG44 & 330 & $13.47 / 76.1-14.13 / 76.1$ & $0.1770-0.1856$ & $\sim 0.1771$ & $\sim 1.0$ & $\sim 4.3$ \\
\hline NGC253 & 10.8 & $13.69 / 76.1-13.91 / 76.1$ & $0.1812-0.1815$ & 0.1815 & 0.042 & $\sim-0.1$ \\
\hline
\end{tabular}

Or still, with the mean density of the structure:

$$
a_{0}=\frac{4 \pi G \rho r}{3}+\frac{\Lambda c^{2} r}{3}+\frac{\Lambda^{2} c^{4} r}{48 \pi G \rho}=\frac{r}{3}\left(4 \pi G \rho+\Lambda c^{2}+\frac{\Lambda^{2} c^{4}}{16 \pi G \rho}\right)
$$

First, constant $a_{0}$ is not independent of time. Indeed, it varies with the age of the universe via the cosmological constant, radius, and mass of the structure. Hence, when the value for $a_{0}$ is adjusted, or selected, those three parameters are fixed. However, we know that the value of $\Lambda$ is time dependent, so that the choice of $r$ and $M$, in particular, fix the value of $\Lambda$, or the mean formation time of the structure. Selecting a typical mass and typical radius for a galaxy is easy (e.g. $10^{10} M_{\odot}$ and $r=40[\mathrm{kpc}]$ ). For smaller structures, $r \rightarrow 0$, the last two terms tend towards zero, which brings us back to Newton's theory:

$$
\begin{gathered}
\frac{a^{2}}{a_{0}}=\frac{G M}{r^{2}} \\
a=\sqrt{a_{0} \frac{G M}{r^{2}}}=\frac{G M}{r^{2}} \text { and } v=\sqrt{\frac{G M}{r}}
\end{gathered}
$$

If $\mathrm{r}$ increases, the final term becomes dominant, or:

$$
a_{0} \rightarrow \frac{\Lambda^{2} c^{4} r^{4}}{36 G M}=\frac{\Lambda^{2} c^{4} r}{48 \pi G \rho} \rightarrow 3.36 \times 10^{42} \Lambda^{2} \frac{r^{4}}{M}
$$

With:

$$
a=\frac{\Lambda c^{2} r}{6} \text { and } v=\frac{\sqrt{\Lambda}}{\sqrt{6}} c r
$$

By selecting a typical mass and radius, a specific value for $a_{0}$ through time can be obtained, knowing that the cosmological constant will vary [38]. Randriamampandry et al. use the MOND theory for the study of the rotation of 15 galaxies and they mention the need to vary the constant $a_{0}$ in order to adjust the rotation curves $\left(a_{0} \sim 0.34\right.$ to $\left.2 \times 10^{-10}\left[\mathrm{~m} \cdot \mathrm{s}^{-2}\right]\right)$. Figure 16 shows the values of $a_{0}$ for three typical masses, $M\left(10^{9}, 10^{10}\right.$ and $\left.10^{11} M_{\odot}\right)$, and radii, $r$ (20 and 40 
$[\mathrm{kpc}])$.

Note that the selected value for $a_{0}\left(\sim 1.2 \times 10^{-10}\left[\mathrm{~m} \cdot \mathrm{s}^{-2}\right]\right)$ corresponds to the formation periods of structures from about 0.32 [Gy] to 1 [Gy] (Figure 17), showing that the MOND theory, in the context of this model, assumes that the galaxies were formed during that $\sim 600$ [My] period, so a short period of time also, but later than advanced in this model, in which formation starts around $200[\mathrm{My}](\mathrm{MW})$.

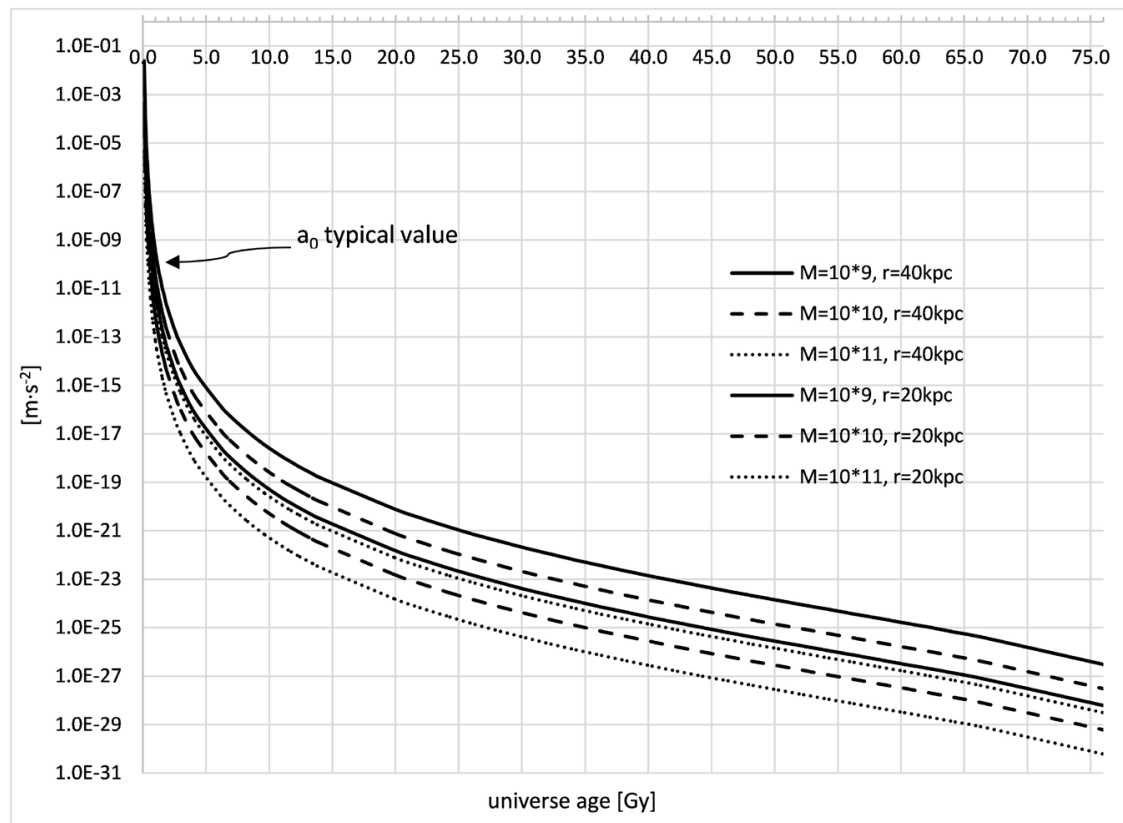

Figure 16. MOND, variation of constant $a_{0}$ with time or $\Lambda(r=20$ and $40[\mathrm{kpc}])$.

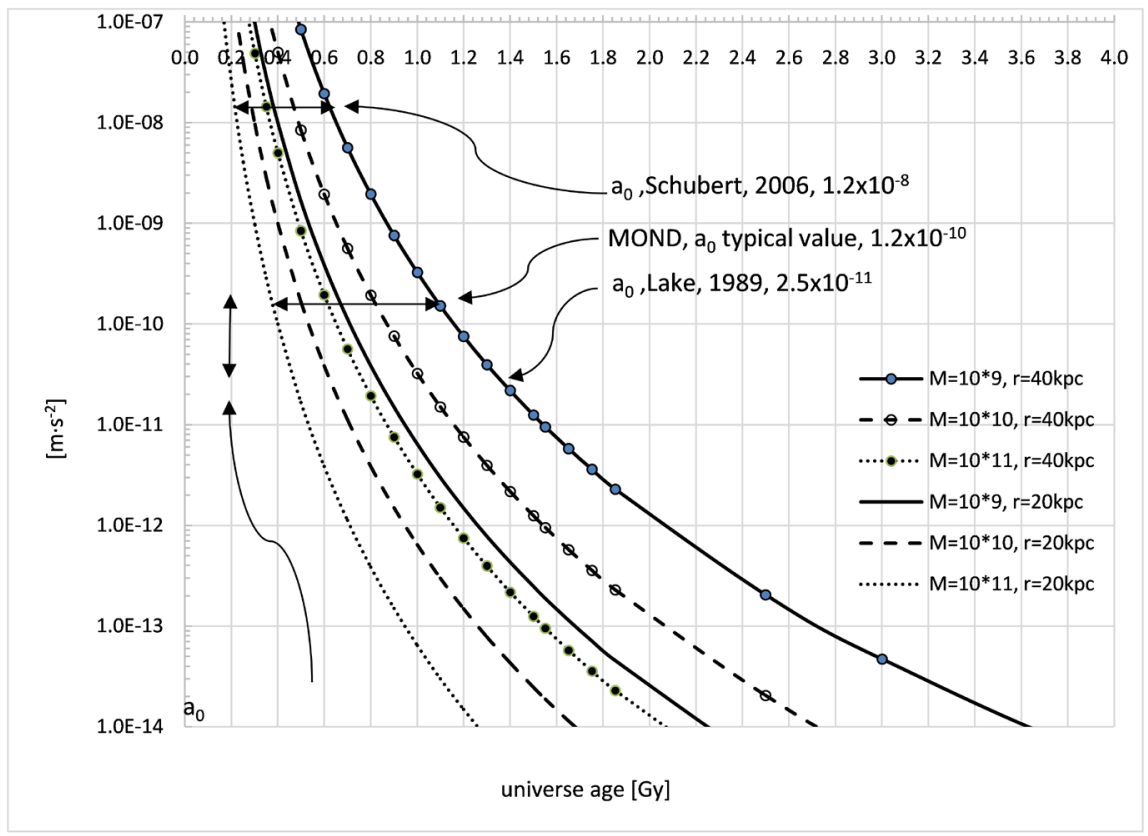

Figure 17. Variation of constant $a_{0}$, MOND with time or $\Lambda(r=20$ and 40 [kpc] and $M=$ $10^{9}$ to $\left.10^{11} M_{\odot}\right)$. 
However, [39] Lake and [40] Schuberth used lower and higher values for $a_{0}$ $\left(2.5 \times 10^{-11}\left[\mathrm{~ms}^{-2}\right], 1.2 \times 10^{-8}\left[\mathrm{~m} \cdot \mathrm{s}^{-2}\right]\right)$ in an effort to explain the rotations of six galaxies (Lake) and one globular cluster NGC4636 (Schuberth), which could mean earlier or later formation times, as early as $\sim 200$ [My] and as late as 450 [My] (Figure 17). Finally, we can see that the MOND theory provides very good models of galaxy rotation, as it relies on the constant $a_{0}$, which in turn relies on the cosmological constant, $\Lambda$, which represents the cosmological gravity, $F_{\Lambda}$, required to explain the rotation speeds of galaxies. However, the MOND theory does not question the value of $G$ or Newton's gravity theory. Indeed, for a cosmological constant value of zero, the Newtonian case is obtained, regardless of mass and radius.

The adapted Newton gravitation constant $G^{\Lambda}$ to take into account this cosmological force of a structure mass $M$ and radius $\mathrm{r}$ by substituting $G$ with the adapted one.

$$
G^{\Lambda}(H)=\left(1+\frac{\Gamma H^{4} r^{3}}{M}\right) G
$$

With: $\Gamma=\frac{c^{2} k_{\Lambda}}{6 G} \sim 6.47 \times 10^{43}\left[\mathrm{~kg} \cdot \mathrm{s}^{4} \cdot \mathrm{m}^{-3}\right]$

The model proposes a very small modification of the $G$ value which depends mainly on the $r^{3}$ size of the structure in question and time of formation (or $H$ ). This small change in $G$ could be a part of the search for a new metric $f(R)$ gravity theory models. A large number of $f(R)$ gravity models have been proposed to explain different cases where the GR theory appears to be less accurate in predicting observations. In a near futur, the observations and measurments of gravitational waves $\mathrm{GW}$ with the development of more sensitive sensors will determine whether or not the GR theory will be a definitive, or not, theory of gravity as it has been formulated in 1916 [41].

\section{Conclusion}

The model proposed herein sheds light on the importance of the cosmological constant, $\Lambda$, which acts as a dominant gravitational force in the early universe. The cosmological constant, referred to by Einstein, is used as the source of the electromagnetic energy of the universe rather than as an effect of opposition to gravity. The model does not consider the existence of energy other than photons. In other words, the notion of dark energy, dark matter (non-baryonic) is not specifically addressed in the model, although the existence of some baryonic dark matter is accepted. The model can partially describe the rotation of certain galaxies without recourse to dark matter (halo), but rather uses the cosmological gravity effect, which has a heavy impact during the early formation period. The galaxies studied herein appear very early in the model, or within the first billion years. In fact, recent observations [42] show the possibility that a star, J0815+4729, had already formed in the MW as early as 200 [My]. Moreover, recent observations of dwarf galaxy MACS1149-JD1 and its star population show 
that the galaxy already existed at $\sim 500[\mathrm{My}]$, and that it had already started to form as early as $\sim 160-200$ [My] after the beginning [9] [43]. Another galaxy detected at $z=6027$ already has a population of stars aged 800 [My] $(z \sim 18)$ or $\sim 200$ [My] after the beginning [44]. Cosmological gravity is behind such early formation, prior to the accepted normal period of a few billion years. Of course, this does not exclude the relative activity of galaxies thereafter (accumulations, collisions, amalgamations, breakups). Finally, in the context of this model, which uses the cosmological constant, the value of constant $a_{0}$ of the MOND theory is more fundamentally explained, allowing to highlight the fact that the theory is an explicit form of cosmological gravity acting on the formation of galaxies. Constant $\mathrm{a}_{0}$ is not fundamentally a constant, and it does not question Newton's law of gravity for great structures. Finally, the model described herein seems interesting for several reasons, but further development is required before its foundations can be validated (complete particle generation, atoms, fusion, etc.). The model is still one among many, fine tuning and improvements are to be expected.

\section{Funding Statement}

Funding for this article was supported by the University of Quebec at Chicoutimi.

\section{Acknowledgements}

The author would like to thank the members of his family, especially his spouse (Danielle) who with patience to bear this work as well his children (Pierre-Luc, Vincent, Claudia), for their encouragement to persevere despite the more difficult periods. Also, a big thank to Mrs. Nadia Villeneuve of UQAC who has prepared the article and references in an acceptable version. Finally, thanks to the University of Quebec at Chicoutimi and to the colleagues of the Department of Applied Sciences for their supports in the realization of this work.

\section{Conflicts of Interest}

The author declares no conflicts of interest regarding the publication of this paper.

\section{References}

[1] North, P. and Observatoire de Sauverny (2011) Astrophysique III: Dynamique stellaire et galactique. École Polytechnique Fédérale de Lausanne, Lausanne.

[2] Martig, M., Bournaud, F. and Teyssier, R. (2009) Morphological Quenching of Star Formation: Making Early-Type Galaxies Red. The Astrophysical Journal, 707, 250. https://doi.org/10.1088/0004-637X/707/1/250

[3] Gessner, E. (1992) Cosmological Constant and the Flat Rotation Curves of Galaxies. Astrophysics and Space Science, 194, 197. https://doi.org/10.1007/BF00643990

[4] Karachentsev, I.D. and Kashibadze, O.G. (2006) Masses of the Local Group and of the M81 Group Estimated from Distortions in the Local Velocity Field. Astrophys- 
ics, 49, 3. https://doi.org/10.1007/s10511-006-0002-6

[5] Peñarrubia, J., Ma, Y.-Z., Walker, M.G. and McConnachie, A. (2014) A Dynamical Model of the Local Cosmic Expansion. Monthly Notices of the Royal Astronomical Society, 443, 2204. https://doi.org/10.1093/mnras/stu879

[6] Blitz, L., Fich, M., et al. (2006) The Galactic Rotation Curve to $\mathrm{r}=18 \mathrm{Kpc}$. Symposium-International Astronomical Union, Vol. 87, Interstellar Molecules, 213-220. https://doi.org/10.1017/S0074180900072582

[7] Natarajan, P., Pacucci, F., Ferrara, A., et al. (2017) Unveiling the First Black Holes with JWST: Multi-Wavelength Spectral Predictions. The Astrophysical Journal, 838, 117. https://doi.org/10.3847/1538-4357/aa6330

[8] Salmon, B., Coe, D., Bradley, L., et al. (2018) RELICS: A Candidate $z \sim 10$ Galaxy Strongly Lensed into a Spatially Resolved Arc. The Astrophysical Journal Letters, 864, L22. https://doi.org/10.3847/2041-8213/aadc10

[9] Hashimoto, T., Laporte, N., Mawatari, K., et al. (2018) The Onset of Star Formation 250 Million Years after the Bing Bang. Nature, 557, 392-395.

https://doi.org/10.1038/s41586-018-0117-Z

[10] Le Fèvre, O., Béthermin, M., Faisst, A., et al. (2020) The ALPINE-ALMA [CII] Survey Strategy, Observations, and Sample Properties of 118 Star-Forming Galaxies at $4<z<6$. Astronomy \& Astrophysics, 643, A1.

[11] Frebel, A., Christlieb, N., Norris, J.E., et al. (2008) Discovery of HE 1523-0901, a Strongly $r$-Process-Enhanced Metal-Poor Star with Detected Uranium. The Astrophysical Journal, 660, L117. https://doi.org/10.1086/518122

[12] Corbelli, E. (2003) Dark Matter and Visible Baryons in M33. Monthly Notices of the Royal Astronomical Society, 342, 199-207. https://doi.org/10.1046/j.1365-8711.2003.06531.x

[13] Giovanelli, R., Haynes, M.P., Rubin, V.C. and Ford, W.K. (1986) UGC 12591: The Most Rapidly Rotating Disk Galaxy. The Astrophysical Journal, 301, L7. https://doi.org/10.1086/184613

[14] Xinyu, D., Michael, E.A., Joel, N.B. and Jon, M.M. (2012) XMM-Newton Detects a Hot Gaseous Halo in the Fastest Rotating Spiral Galaxy UGC 12591. The Astrophysical Journal, 755, 107. https://doi.org/10.1088/0004-637X/755/2/107

[15] Gentile, G., Józsa, G.I.G., Serra, P., et al. (2013) HALOGAS: Extraplanar Gas in NGC 3198. Astronomy \& Astrophysics, 554, A125. https://doi.org/10.1051/0004-6361/201321116

[16] Pence, W.D. (1981) A Photometric and Kinematic Study of the Barred Spiral Galaxy NGC 253. II. The Velocity Field. The Astronomical Journal, 247, 473-483. https://doi.org/10.1086/159056

[17] Côté, S., Carignan, C. and Freeman, K.C. (2000) The Various Kinematics of Dwarf Irregular Galaxies in Nearby Groups and Their Dark Matter Distributions. The Astronomical Journal, 120, 3027-3059. https://doi.org/10.1086/316883

[18] Van Dokkum, P., Abraham, R., Brodie, J., et al. (2016) A High Stellar Velocity Dispersion and $\sim 100$ Globular Clusters for the Ultra-Diffuse Galaxy Dragonfly 44 . The Astrophysical Journal Letters, 828, L6. https://doi.org/10.3847/2041-8205/828/1/L6

[19] Zwicky, F. (1937) On the Masses of Nebulae and of Clusters of Nebulae, The Astrophysical Journal, 86, 217-246. https://doi.org/10.1086/143864

[20] Mayall, N.U. (1960) Advantages of Electronic Photography for Extragalactic Spectroscopy. Annales d Astrophysique, 23, 344-370. 
[21] Van Albada, G.B. (1961) Evolution of Clusters of Galaxies under Gravitational Forces. The Astronomical Journal, 66, 590. https://doi.org/10.1086/108469

[22] Omer Jr., G.C., Page, T.L. and Wilson, A.G. (1965) The Coma Cluster of Galaxies. I. Size and Structure. The Astronomical Journal, 70, 440.

https://doi.org/10.1086/109759

[23] Peebles, P.J.E. (1970) Structure of the Coma Cluster of Galaxies. The Astronomical Journal, 75, 13-20. https://doi.org/10.1086/110933

[24] Rood, H.J., Page, T.L., Kintner, E.C. and King, I.R. (1972) The Structure of the Coma Cluster of Calaxies. The Astrophysical Journal, 175, 627-647.

https://doi.org/10.1086/151585

[25] Kent, S.M. and Gunn, J.E. (1982) The Dynamics of Rich Clusters of Galaxies. I, the Coma Cluster. The Astronomical Journal, 87, 945-971. https://doi.org/10.1086/113178

[26] Merritt, D. (1987) The Distribution of Dark Matter in the Coma Cluster. The Astrophysical Journal, 313, 121-135. https://doi.org/10.1086/164953

[27] White, S.D.M., Navarro, J.F., Evrard, A.E. and Frenk, C.S. (1993) The Baryon Content of Galaxy Clusters: A Challenge to Cosmological Orthodoxy. Nature, 366, 429-433. https://doi.org/10.1038/366429a0

[28] Gavazzi, R., Adami, C., Durret, F., et al. (2009) A Weak Lensing Study of the Coma Cluster. Astronomy \& Astrophysics, 498, L33-L36.

https://doi.org/10.1038/366429a0

[29] Godwin, J.G. and Peach, J.V. (1977) Studies of Rich Clusters of Galaxies IV. Photometry of the Coma Cluster. Monthly Notices of the Royal Astronomical Society, 181, 323-337. https://doi.org/10.1038/366429a0

[30] Abell, G.O. (1965) Clustering of Galaxies. Annual Review of Astronomy and Astrophysics, 3, 1-22. https://doi.org/10.1146/annurev.aa.03.090165.000245

[31] Abell, G.O. (1977) The Luminosity Function and Structure of the Coma Cluster. The Astrophysical Journal, 213, 327. https://doi.org/10.1086/155159

[32] Chincarini, G. and Rood, H.J. (1975) Size of the Coma Cluster. Nature, 257, 294-295. https://doi.org/10.1038/257294a0

[33] Wang, T., Elbaz, D., Daddi, E., et al. (2016) Discovery of a Galaxy Cluster with a Violently Starbursting Core at $z=2.506$. The Astrophysical Journal, 828, 56. https://doi.org/10.3847/0004-637X/828/1/56

[34] Wang, T., Schreiber, C., Elbaz, D. (2019) A Dominant Population of Optically Invisible Massive Galaxies in the Early Universe. Nature, 572, 211-214.

https://doi.org/10.1038/s41586-019-1452-4

[35] Brownstein, J.R. and Moffat, J.W. (2006) Galaxy Rotation Curves without Nonbaryonic Dark Matter. The Astrophysical Journal, 636, 721-741. https://doi.org/10.1086/498208

[36] Bouwens, R.J., Illingworth, G.D., et al. (2006) Rapid Evolution of the Most Luminous Galaxies during the First 900 Million Years. Nature, 443, 189-192. https://doi.org/10.1038/nature05156

[37] Zhou, Y., Zhao, Z.-C. and Chang, Z. (2017) Searching for a Cosmological Preferred Direction with 147 Rotationally Supported Galaxies. The Astrophysical Journal, 847, 86. https://doi.org/10.3847/1538-4357/aa8991

[38] Randriamampandry, T.H. and Carignan, C. (2014) Galaxy Mass Models: MOND versus Dark Matter Haloes. Monthly Notices of the Royal Astronomical Society, 439, 2132-2145. https://doi.org/10.1093/mnras/stu100 
[39] Lake, G. (1989) Testing Modifications of Gravity. The Astrophysical Journal, 345, L17-L19. https://doi.org/10.1086/185541

[40] Schuberth, Y., et al. (2006) Dynamics of the NGC 4636 Globular Cluster System. In: Stanghellini, L., Walsh, J.R. and Douglas, N.G., Eds., Planetary Nebulae beyond the Milky Way, ESO Astrophysics Symposia European Southern Observatory, Springer, Berlin.

[41] Corda, C. (2009) Interferometric Detection of Gravitational Waves: The Definitive Test for General Relativity. International Journal of Modern Physics D, 18, 22752282. https://doi.org/10.1086/185541

[42] Aguado, D.S., et al. (2018) J0815+4729: A Chemically Primitive Dwarf Star in the Galactic Halo Observed with Gran Telescopio Canarias. The Astrophysical Journal Letters, 852, L20. https://doi.org/10.3847/2041-8213/aaa23a

[43] Hoag, A., Bradač, M., Brammer, G., et al. (2018) HST Grism Observations of a Gravitationally Lensed Redshift 9.5 Galaxy. The Astrophysical Journal, 854, 39. https://doi.org/10.3847/1538-4357/aaa9c2

[44] Richard, J., Kneib, J.-P., Ebeling, H., et al. (2011) Discovery of a Possibly Old Galaxy at $z=6.027$, Multiply Imaged by the Massive Cluster Abell 383. Monthly Notices of the Royal Astronomical Society Letters, 414, L31.

https://doi.org/10.1111/j.1745-3933.2011.01050.x 